\title{
MYB Gene Family in Potato (Solanum tuberosum L.): Genome-Wide Identification of Hormone-Responsive Reveals Their Potential Functions in Growth and Development
}

\author{
Wenjun Sun ${ }^{1,2,+}$, Zhaotang Ma ${ }^{1,2,+}$, Hui Chen ${ }^{2}$ and Moyang Liu ${ }^{1, *(D)}$ \\ 1 School of Agriculture and Biology, Shanghai Jiao Tong University, Shanghai 200240, China; \\ sunnan82475@163.com (W.S.); WaohUncle_Ma@163.com (Z.M.) \\ 2 College of Life Science, Sichuan Agricultural University, Ya'an 625014, China; chenhui@sicau.edu.cn \\ * Correspondence: cooljeep@sjtu.edu.cn; Tel.: +86-1719-1207-171 \\ + These authors contributed equally to this work.
}

Received: 6 August 2019; Accepted: 27 September 2019; Published: 29 September 2019

\begin{abstract}
As an important nongrain crop, the growth and yield of potato (Solanum tuberosum L.) is often affected by an unfavorable external environment in the process of cultivation. The MYB family is one of the largest and most important gene families, participating in the regulation of plant growth and development and response to abiotic stresses. Several MYB genes in potato that regulate anthocyanin synthesis and participate in abiotic stress responses have been identified. To identify all Solanum tuberosum L. MYB (StMYB) genes involved in hormone or stress responses to potentially regulate potato growth and development, we identified the MYB gene family at the genome-wide level. In this work, 158 StMYB genes were found in the potato genome. According to the amino acid sequence of the MYB domain and gene structure, the StMYB genes were divided into R2R3-MYB and R1R2R3-MYB families, and the R2R3-MYB family was divided into 20 subgroups (SGs). The expression of 21 StMYB genes from different SGs in roots, stems, leaves, flowers, shoots, stolons, young tubers, and mature tubers was determined by quantitative real-time polymerase chain reaction (qRT-PCR). The expression patterns of $S t M Y B$ genes in potatoes treated with abscisic acid (ABA), indole-3-acetic acid (IAA), gibberellin acid 3 (GA3), NaCl, mannitol, and heat were also measured. We have identified several potential candidate genes that regulate the synthesis of potato flavonoids or participate in hormone or stress responses. This work provides a comprehensive understanding of the MYB family in potato and will lay a foundation for the future investigation of the potential functions of $S t M Y B$ genes in the growth and development of potato.
\end{abstract}

Keywords: hormone or stress responses; potato genome; StMYB; expression patterns

\section{Introduction}

The abiotic stresses that plants encounter in their growth process seriously affect their growth and productivity. At every moment of a plant's life, thousands of genes are expressed in a coordinated manner to regulate its growth and development and help it resist adverse external environments. Identifying the key genes involved in plant responses to abiotic stress and exploring their molecular mechanisms can lay a foundation for the breeding of crops resistant to abiotic stress [1,2]. Transcription factors (TFs) play a crucial role in plant growth and development through self-regulation and regulation of downstream target gene expression [3]. TFs can have a DNA-binding domain, transcriptional activation domain, and dimerization domain and are involved in physiological and biochemical processes that activate or inhibit transcription in response to endogenous and exogenous stimuli. 
The MYB family is the largest and most functional TF family in plants, animals, and fungi [4]. The imperfect repeat $(\mathrm{R})$ is the basic unit of the DNA-binding domain of MYB proteins and consists of approximately 52 amino acid residues. Each repeat has a helix-turn-helix (HTH) structure motif, which is composed of three conserved tryptophan residues (W) with an interval of 18-19 amino acids. According to the number of Rs contained in each MYB protein, the MYB proteins can be divided into 4R-MYB, R2R3-MYB, R1R2R3-MYB (3R-MYB), and MYB-related proteins [5,6]. 4R-MYB is the smallest class. Each gene in this class contains four R1/R2 repeats, and little is known about these proteins in plants. The second class of the MYB family contains three continuous repeats (R1R2R3-MYB), and 3R-MYB proteins have been found in most eukaryotic genomes, playing a role in cell cycle control [7]. The third class of the MYB family, called MYB-related, contains a single or partial MYB repeat [8-10]. Among these four classes, R2R3-MYB is the largest in plants, and the genes of this family may have evolved from the loss of the R1 repeat in the R1R2R3-MYB gene [11]. However, it has also been suggested that the 3R-MYB gene evolved from the R2R3-MYB gene, which acquired the R1 repeat [12]. There is a highly conserved MYB domain in the structure of R2R3-MYB TFs, and their C-termini are highly variable. R2R3-MYB usually determines the interaction between proteins $[6,13,14]$.

The R2R3-MYB family is widely involved in various physiological activities of plants, such as responding to environmental factors and plant hormones, participating in the regulation of primary and secondary metabolism, regulating cell cycle and cell differentiation, and leaf morphogenesis $[15,16]$. According to the conserved domains of MYB genes and their functions, the R2R3-MYB family in Arabidopsis thaliana (L.) Heynh. is divided into 23 subgroups (SGs) [5]. Each subgroup plays an important role in different physiological and biochemical activities. Members of SG 7 have a conserved GRTxRSxMK motif, which can positively regulate the biosynthesis of flavonoids. AtMYB11, $A t M Y B 12$, and $A t M Y B 111$ in SG 7 were found to be involved in the regulation of flavonoid biosynthesis in Arabidopsis thaliana (L.) Heynh. [17]. Members of SG 5 have a conserved DExWRLxxT motif, and members of SG 6 have a conserved KPRPRFb motif, which can form complexes with members of the WD40 and bHLH families to participate in the biosynthesis of proanthocyanidins and anthocyanins, respectively. It has been found that AtMYB75, AtMYB90, AtMYB113, and AtMYB114 in SG 6 can regulate anthocyanin biosynthesis, while $A t M Y B 123$ in SG 5 can regulate proanthocyanidin synthesis in the seed coat of Arabidopsis thaliana (L.) Heynh. [18,19].

Studies have shown that R2R3-MYB family genes also play an important role in the plant responses to abiotic stress, besides participating in various physiological activities. Several MYB genes involved in stress response have been identified in wheat, among which TaMYB1 can respond to abiotic stress and ABA treatment, TaMYB2A in transgenic Arabidopsis thaliana (L.) Heynh. showed a variety of abiotic stress tolerances, while TaMYB33 can reconstruct the osmotic balance of transgenic plants, thereby improving their salt tolerance and drought tolerance [20-22]. Owing to the powerful function of MYB genes, an increasing number of MYB genes have been identified, and the MYB gene family has been identified from many dicotyledonous and monocotyledonous plants, including Arabidopsis thaliana (L.) Heynh. (197 members) [23], Cucumis sativus L. (55 members) [6], Pyrus bretschneideri Rehd. (184 members) [24], Beta vulgaris L. (70 members) [25], Gossypium hirsutum Linn. (524 members) [26], Oryza sativa L. (155 members) [23], and Zea mays Linn. (158 members) [27] at the genome-wide level.

In the potato cultivation process, abiotic stresses, including drought and high salt, often harm potato growth and lead to a decline in yield. Therefore, it is urgent to explore potato stress resistance genes for potato breeding and production. At present, some $M Y B$ genes involved in stress response have been identified in potato. For example, StMYB1R-1 is involved in activating drought-related genes and improves potato drought tolerance by regulating water loss [28]. In addition, studies have shown that in transgenic potato, the increased expression of IbMYB1 affects secondary metabolism, thereby improving potato tolerance to stress [29]. Li et al. previously identified the MYB gene family from potato, and analyzed the chromosome location, syntenic, and the role of $M Y B$ genes in potato development and stress response [30]. In this study, $158 M Y B$ genes were identified from the potato genome by different identification methods, and genes related to 
growth, development, and stress response were excavated. Their motif composition, intron and exon distribution, and tandem duplication and segmental duplication events were comprehensively analyzed. Their evolutionary relationships with dicotyledonous Arabidopsis thaliana (L.) Heynh., Fagopyrum tataricum Gaertn., Manihot esculenta Crantz, Nicotiana attenuate, Solanum lycopersicum, Vitis vinifera L., and monocotyledonous Oryza sativa L. were compared. At the same time, we measured the expression levels of $S t M Y B$ genes in roots, stems, leaves, flowers, shoots, stolons, young tubers, and mature tubers. More importantly, by determining the expression patterns of $S t M Y B$ genes under ABA, IAA, GA3, NaCl, mannitol, and high temperature treatments, we identified some StMYB genes that may potentially play a vital role in potato growth and hormone stress resistance. This study can provide a meaningful reference for potato breeding improvement.

\section{Results}

\subsection{Identification of the MYB Gene Family in Potato}

We excavated 158 MYB genes from the potato genome and named them StMYB1 to StMYB158 according to their distribution on the chromosomes (Table S1). The coding sequence lengths, molecular weights (MWs), subcellular localizations, and isoelectric points (PIs) of these genes are shown in Table S1. StMYB26 had the smallest amino acid sequence of 53 aa, while StMYB146 had the largest amino acid sequence of 1021 aa. The MWs of these StMYB proteins ranged from $5.89 \mathrm{kDa}$ (StMYB26) to $113.39 \mathrm{kDa}$ (StMYB146), and their PIs ranged from 4.59 (StMYB78) to 10.26 (StMYB10). StMYB140 was located in chloroplasts; StMYB10, StMYB26, StMYB37, and StMYB138 were located in mitochondria; and other StMYB proteins were located in the nucleus (Table S1).

\subsection{Phylogenetic Tree Analysis of MYB Genes in Potato and Arabidopsis thaliana (L.) Heynh}

We constructed a maximum likelihood (ML) phylogenetic tree with 158 StMYB proteins and 128 MYB proteins from Arabidopsis thaliana (L.) Heynh. using Mega 7.0. Five of 128 MYB genes in Arabidopsis thaliana (L.) Heynh. belonged to the 3R-MYB subfamily, and the others belonged to the R2R3-MYB subfamily. The R2R3-MYB subfamily was further divided into S 1, S 2, S 3, S 4, S 5, S 6, S 7, S 9, S 10, S 11, S 12, S 13, S 14, S 15, S 17, S 18, S 19, S 20, S 21, S 22, S 23, S 24, and S 25. From the phylogenetic tree, we can see that three StMYB genes belonged to the 3R-MYB subfamily (StMYB116, StMYB121, and StMYB146), while 155 R2R3-MYB subfamily genes belonged to S 1 (8 members), S 2 (6 members), S 3 ( 2 members), S 4 (8 members), S 6 ( 2 members), S 7 (6 members), S 9 (7 members), S 11 (4 members), S 13 (10 members), S 14 (24 members), S 15 (6 members), S 17 (7 members), S 18 (7 members), S 19 (1 member), S 20 (30 members), S 21 (4 members), S 22 (15 members), S 23 (2 members), S 24 (3 members), and S 25 (3 members) subgroups (Figure 1). There were no StMYB genes in S 5, S 10, or $\mathrm{S} 12$. 


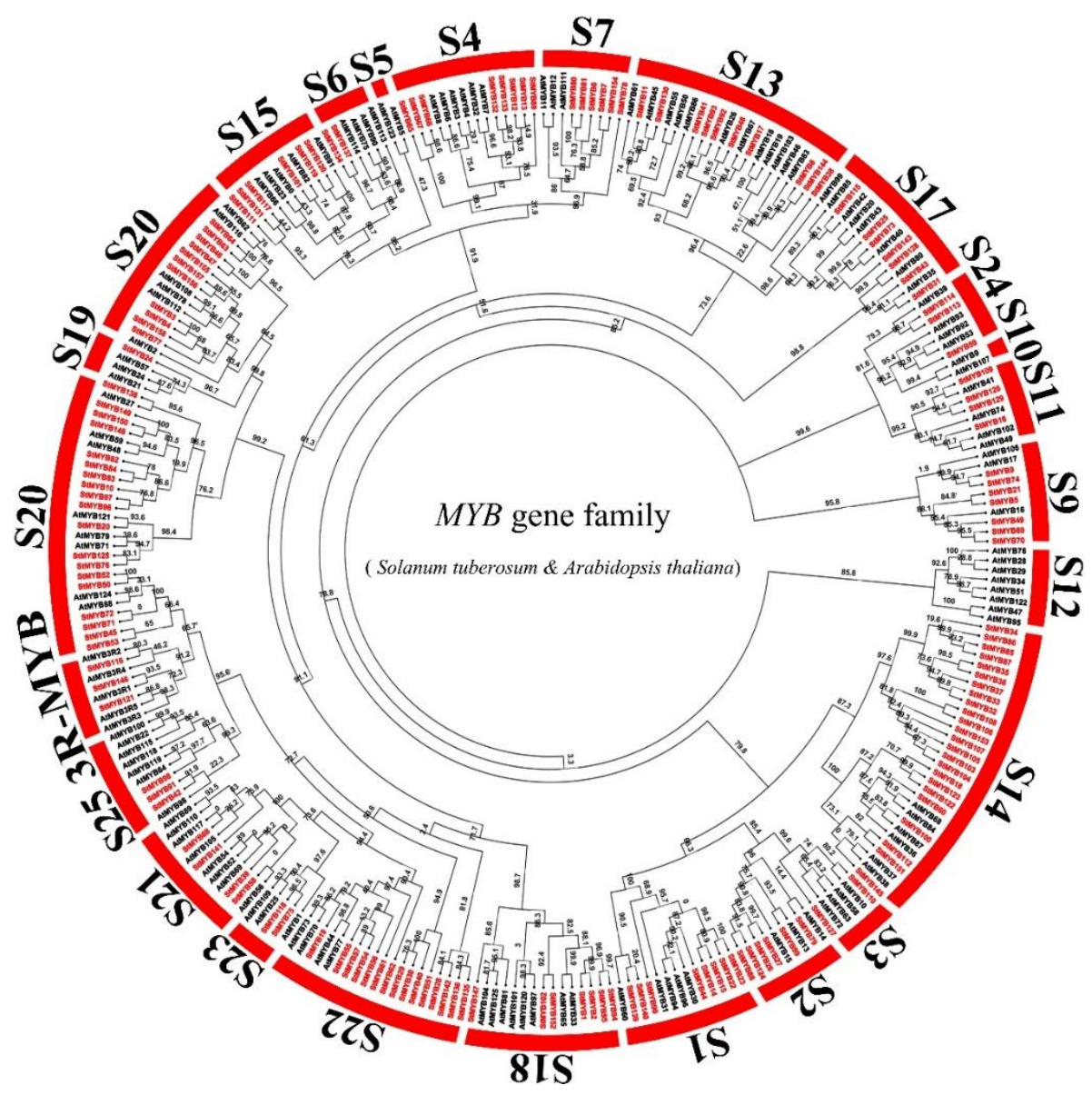

Figure 1. Unrooted phylogenetic tree representing the relationships among 158 MYB genes of potato and Arabidopsis thaliana (L.) Heynh. with 1000 bootstrap replicates by the maximum likelihood (ML) method. MYB genes from potato and Arabidopsis thaliana (L.) Heynh. are classified into S1, S2, S3, S4, $\mathrm{S} 5, \mathrm{~S} 6, \mathrm{~S} 7, \mathrm{~S} 9, \mathrm{~S} 10, \mathrm{~S} 11, \mathrm{~S} 12, \mathrm{~S} 13, \mathrm{~S} 14, \mathrm{~S} 15, \mathrm{~S} 17, \mathrm{~S} 18, \mathrm{~S} 19, \mathrm{~S} 20, \mathrm{~S} 21, \mathrm{~S} 22, \mathrm{~S} 23, \mathrm{~S} 24$, and S25. The genes in potato are marked in red, while those in Arabidopsis thaliana (L.) Heynh. are marked in black.

\subsection{Gene Structure, Sequence Composition, and Promoter Cis-Acting Element Analysis}

The phylogenetic tree was constructed from the amino acid sequences of 158 StMYB genes, and the R2R3-MYB family genes were still divided into 20 subgroups (Figure 2A). Figure 2B shows that all 158 StMYB genes contained a Myb DNA-binding domain and 1 to 10 introns. Some StMYB genes had a large number of introns, including StMYB116 (6 introns), StMYB121 (7 introns), and StMYB146 (10 introns) from the 3R-MYB family and StMYB45 (5 introns), StMYB50 (9 introns), StMYB51 (7 introns), StMYB52 (10 introns), StMYB71 (7 introns), and StMYB72 (8 introns) in $S 20$. The other StMYB genes had 1 to 3 introns, and the number of introns of $S t M Y B$ genes in the same subgroup was generally similar.

By detecting the motif composition of StMYB proteins characteristic regions, it was found that StMYB51, StMYB135, StMYB136, StMYB10, StMYB28, StMYB36, StMYB33, and StMYB32 only contain motif 1 (Figure 2C). Proteins containing only motif 3 included StMYB40, StMYB30, StMYB29, StMYB142, and StMYB54. StMYB67 and StMYB132 only contained motifs 1 and 6; StMYB80 only contained motifs 1 and 2; StMYB4 only contained motifs 3 and 5; StMYB155 and StMYB63 only contained motifs 1 and 7; StMYB53 and StMYB57 only contained motifs 2 and 3; and proteins containing only motifs 1 and 8 included StMYB84, StMYB83, StMYB82, StMYB96, StMYB97, StMYB148, and StMYB150. Almost all the other proteins contained motifs 2 and 3 . Only the proteins of $S 1$ contained motif 9 , while those of $\mathrm{S} 9$ contained motif 10. In conclusion, most StMYB proteins from the same subgroup had similar motif compositions (Figure 2C). 


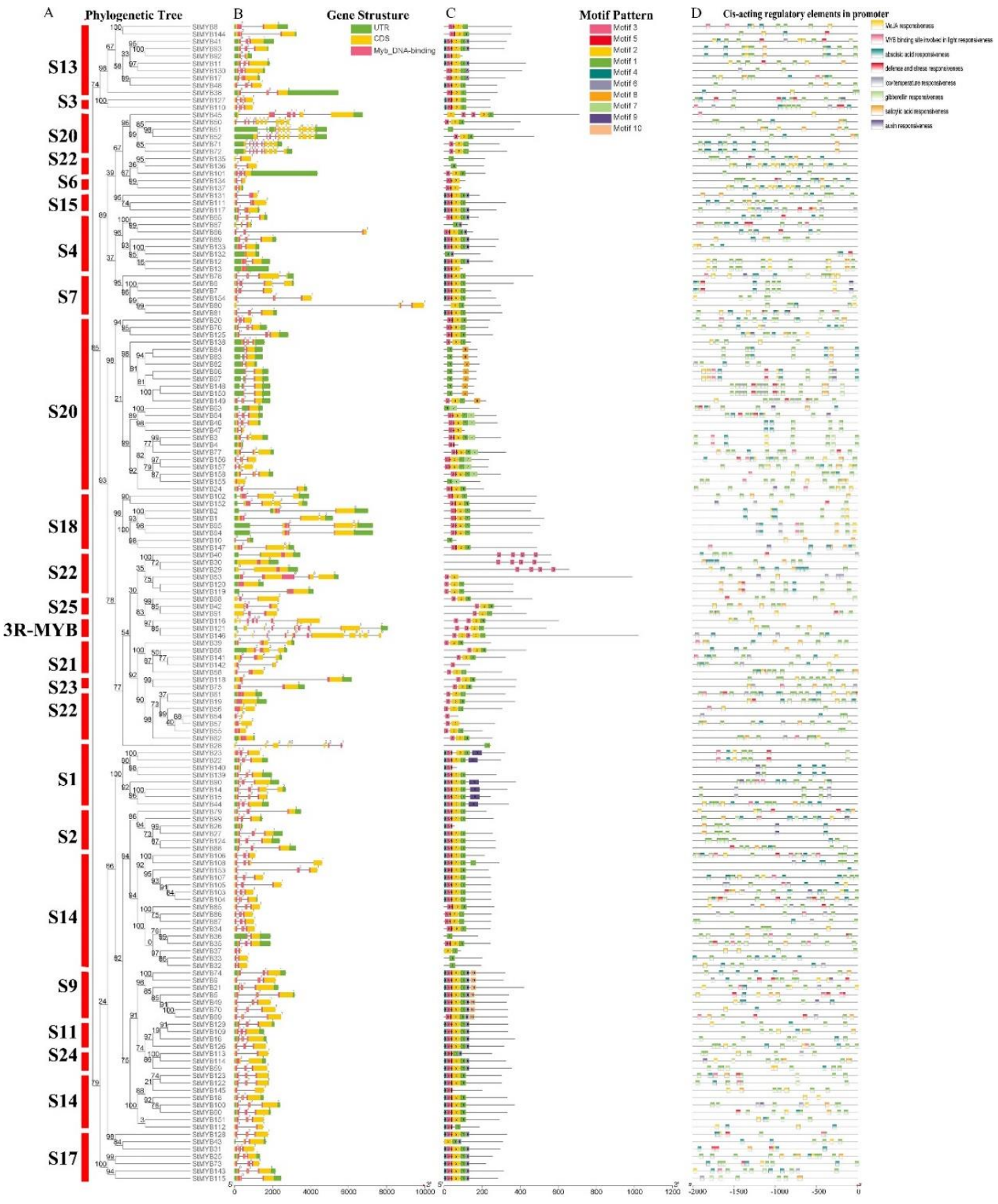

Figure 2. Phylogenetic relationships, gene structures, architectures of the conserved protein motifs, and cis-acting elements analysis of the $M Y B$ genes from potato. (A) The phylogenetic tree was constructed based on the full-length sequences of potato MYB proteins using Geneious R11 software. (B) Exon-intron structures of potato $M Y B$ genes. Blue boxes indicate untranslated $5^{\prime}$ - and $3^{\prime}$-regions, and black lines indicate introns. The number indicates the phases of the corresponding introns. (C) The motif compositions of the potato MYB proteins. The motifs, numbered 1-10, are displayed in different colored boxes. The sequence information for each motif is provided in Table S2. The protein length can be estimated using the scale at the bottom. (D) The cis-acting elements analysis of StMYB genes promoters. Blocks of different colors represent light responsiveness elements, low temperature responsiveness elements, salicylic acid responsiveness elements, abscisic acid responsiveness elements, MeJA responsiveness elements, auxin responsiveness elements, gibberellin responsiveness elements, and defense and stress responsiveness elements. 
The promoter prediction results showed that the cis-acting elements in the promoter region of StMYB genes included MeJA responsiveness elements, MYB-binding sites that were part of light responsiveness elements, $\mathrm{ABA}$ responsiveness elements, defense and stress responsiveness elements, low temperature responsiveness elements, gibberellin acid (GA) responsiveness elements, salicylic acid (SA) responsiveness elements, and auxin responsiveness elements (Figure 2D). These cis-acting elements could be classified into three categories, including phytohormones, environmental stress, and photoresponsive elements (Figure 2D). The GA responsiveness elements were most common in the StMYB gene promoters, which were present in 149 promoters. There were 113 StMYB gene promoters containing ABA responsiveness elements, 87 StMYB gene promoters containing MeJA responsiveness elements, 72 StMYB gene promoters containing SA responsiveness elements, 49 StMYB gene promoters containing defense and stress responsiveness elements, 46 StMYB gene promoters containing light responsiveness elements, 41 StMYB gene promoters containing low temperature responsiveness elements, and $24 S t M Y B$ gene promoters containing auxin responsiveness elements. In general, phytohormone responsiveness elements other than auxin responsiveness elements were most common in StMYB gene promoters (Figure 2D).

\subsection{Analysis of Chromosome Distribution, Tandem Gene Duplication, and Segmental Gene Duplication of StMYB Genes}

The 158 StMYB genes were unevenly distributed on 12 chromosomes (Figure 3). There were 23 StMYB genes on chromosome 3, 20 StMYB genes on chromosome 6, 17 StMYB genes on chromosome 5 and chromosome 10, 14 StMYB genes on chromosome 4, 13 StMYB genes on chromosome 1, 12 StMYB genes on chromosome 2, 11 StMYB genes on chromosome 7, 14 StMYB genes on chromosome 9, chromosome 11 and chromosome 12, and 7 StMYB genes on chromosome 8 (Figure 3). A total of 36 tandem-duplicated gene pairs were unevenly distributed on 12 chromosomes. There were 5 tandem-duplicated gene pairs on chromosome 3 (StMYB26 and StMYB27, StMYB29 and StMYB30, StMYB32 and StMYB33, StMYB35 and StMYB36, StMYB46 and StMYB47) and chromosome 5 (StMYB63 and StMYB64, StMYB65 and StMYB66, StMYB66 and StMYB67, StMYB69 and StMYB70, StMYB71 and StMYB72); 4 tandem-duplicated gene pairs on chromosome 1 (StMYB1 and StMYB2, StMYB3 and StMYB4, StMYB6 and StMYB7, StMYB12 and StMYB13), chromosome 6 (StMYB80 and StMYB81, StMYB83 and StMYB84, StMYB92 and StMYB93, StMYB94 and StMYB95), chromosome 7 (StMYB103 and StMYB104, StMYB104 and StMYB105, StMYB105 and StMYB106, StMYB107 and StMYB108), and chromosome 10 (StMYB132 and StMYB133, StMYB136 and StMYB137, StMYB139 and StMYB140, StMYB141 and StMYB142); 2 tandem-duplicated gene pairs on chromosome 2 (StMYB14 and StMYB15, StMYB22 and StMYB23), chromosome 9 (StMYB119 and StMYB120, StMYB120 and StMYB121), chromosome 11 (StMYB148 and StMYB149, StMYB149 and StMYB150), and chromosome 12 (StMYB156 and StMYB157, StMYB157 and StMYB158); and only one tandem-duplicated gene pair on chromosome 4 (StMYB51 and StMYB52) and chromosome 8 (StMYB113 and StMYB114) (Figure 3).

In addition to tandem-duplicated gene pairs, 41 pairs of segmental-duplicated gene pairs were also found on potato chromosomes (Figure 4). All in all, by analyzing the duplication events in StMYB genes, we found that some $S t M Y B$ genes were produced by tandem duplication and segmental duplication, and these gene duplication events may be one of the drivers of the evolution of StMYB genes. 


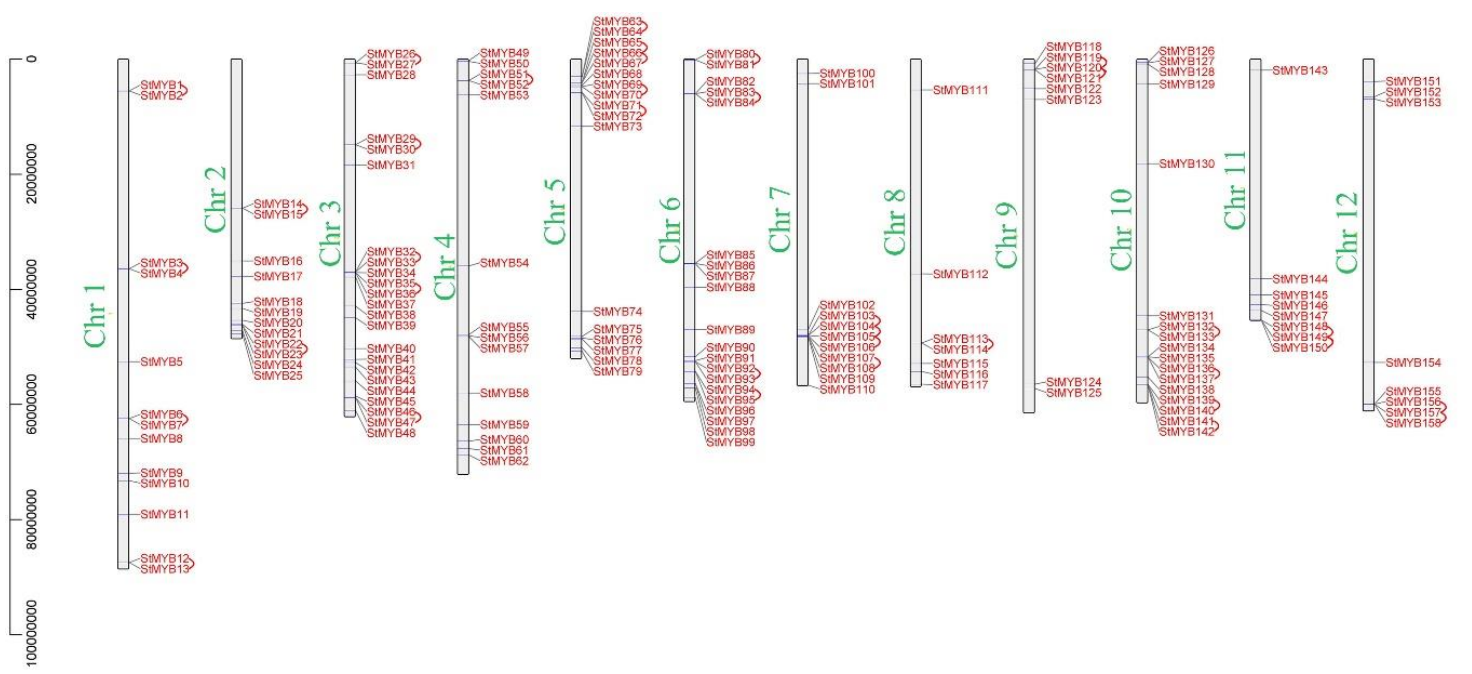

Figure 3. The distribution of $M Y B$ genes on chromosomes. The chromosome number is indicated to the left of each chromosome.

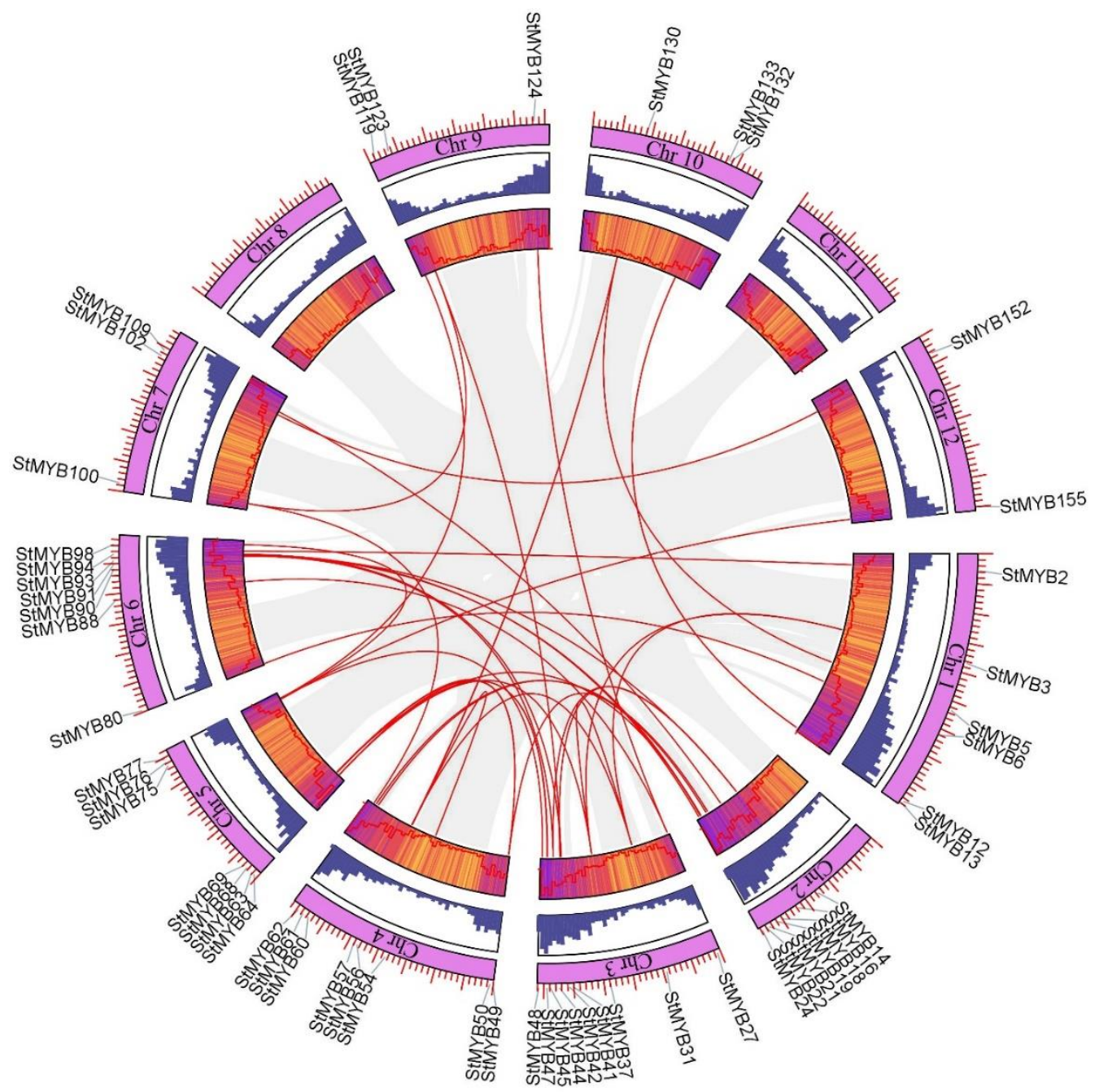

Figure 4. The interchromosomal relationships of the potato MYB genes. Colored lines indicate all syntenic blocks in the potato genome. 


\subsection{Evolutionary Analysis of StMYB Genes and the MYB Genes of Several Different Species}

After exploring the evolutionary relationship of $M Y B$ genes in potato, we constructed a phylogenetic tree including dicotyledonous potato, Arabidopsis thaliana (L.) Heynh., Solanum lycopersicum, Vitis vinifera L., Manihot esculenta Crantz, and monocotyledonous Oryza sativa L. to explore the evolutionary relationship of $M Y B$ genes in different plants (Figure 5). As shown in Figure 5, MYB genes in all plants were still divided into the 3R-MYB subfamily and the R2R3-MYB subfamily, while the R2R3-MYB subfamily was further divided into S 1, S 2, S 3, S 4, S 5, S 6, S 7, S 9, S 11, S 12, S 13, S 14, S 15, S 17, S 18, S 19, S 20, $\mathrm{S} 21, \mathrm{~S} 22, \mathrm{~S} 23, \mathrm{~S} 24$, and S 25. There were no MYB genes from Oryza sativa L. in S 2 or S 3; no StMYB genes in S 5; no MYB genes from potato, Solanum lycopersicum, or Oryza sativa L. in S 10; and only MYB genes from Arabidopsis thaliana (L.) Heynh. and Manihot esculenta Crantz in S 12. In addition to these subgroups, other subgroups included $M Y B$ genes from six plants. Ten motifs were detected in all MYB protein sequences, and most MYB proteins contained motifs 1, 2, 4, and 5, while motifs 6 to 9 only existed in a few MYB proteins. MYB genes of the same subgroup, especially those with similar homology, have similar motif compositions and similar positions, which further supports the reliability of phylogenetic trees.

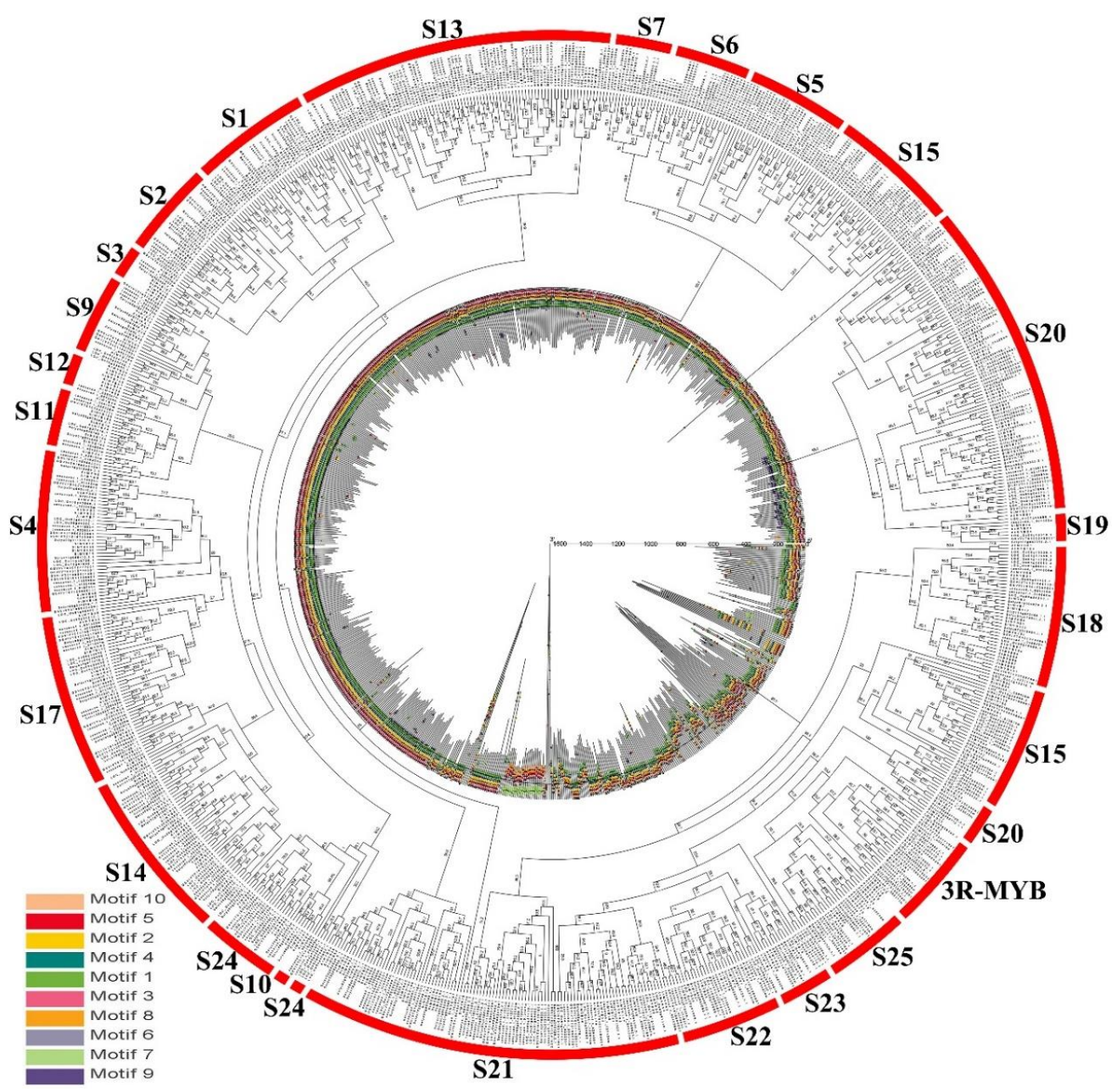

Figure 5. Phylogenetic relationships and motif compositions of the MYB proteins from six different plant species (Solanum tuberosum L., Arabidopsis thaliana (L.) Heynh., Solanum lycopersicum, Vitis vinifera L., Manihot esculenta Crantz, and Oryza sativa L.). MYB genes from multiple species are classified into S 1, S 2, S 3, S 4, S 5, S 6, S 7, S 9, S 11, S 12, S 13, S 14, S 15, S 17, S 18, S 19, S 20, S 21, S 22, S 23, S 24, and $S$ 25. Outer layer: An unrooted ML phylogenetic tree constructed using Mega 7.0. Inner layer: Distribution of the conserved motifs in MYB proteins. The differently colored boxes represent different motifs and their positions in each MYB protein sequence. 
We further constructed a synteny analysis between $M Y B$ genes in potato and $M Y B$ genes in many plants, including dicotyledonous Arabidopsis thaliana (L.) Heynh., Fagopyrum tataricum Gaertn., Manihot esculenta Crantz, Nicotiana attenuate, Solanum lycopersicum, Vitis vinifera L., and monocotyledonous Oryza sativa L. (Figure 6). The StMYB genes were homologous to genes in other plants, and syntenic conservation was observed among Solanum lycopersicum (159 orthologous gene pairs dispersed on all chromosomes), Manihot esculenta Crantz (159 orthologous gene pairs dispersed on all chromosomes), Vitis vinifera L. (106 orthologous gene pairs dispersed on all chromosomes except chromosome 10), Fagopyrum tataricum Gaertn. (70 orthologous gene pairs dispersed on all chromosomes), Arabidopsis thaliana (L.) Heynh. (65 orthologous gene pairs dispersed on all chromosomes), Nicotiana attenuate (21 orthologous gene pairs dispersed on all chromosomes except chromosome 4 and chromosome 9), and Oryza sativa L. (21 orthologous gene pairs dispersed on all chromosomes except chromosome 3 and chromosome 12) (Figure 6). The results of the syntenic analysis showed that StMYB44 formed homologous gene pairs with $M Y B$ genes in different plants, including five pairs of homologous gene pairs with $M Y B$ genes in Solanum lycopersicum, three pairs of homologous gene pairs with MYB genes in Manihot esculenta Crantz, and two pairs of homologous gene pairs with MYB genes in Vitis vinifera L., which indicated that StMYB44 may have played a key role in evolution (Table S3).

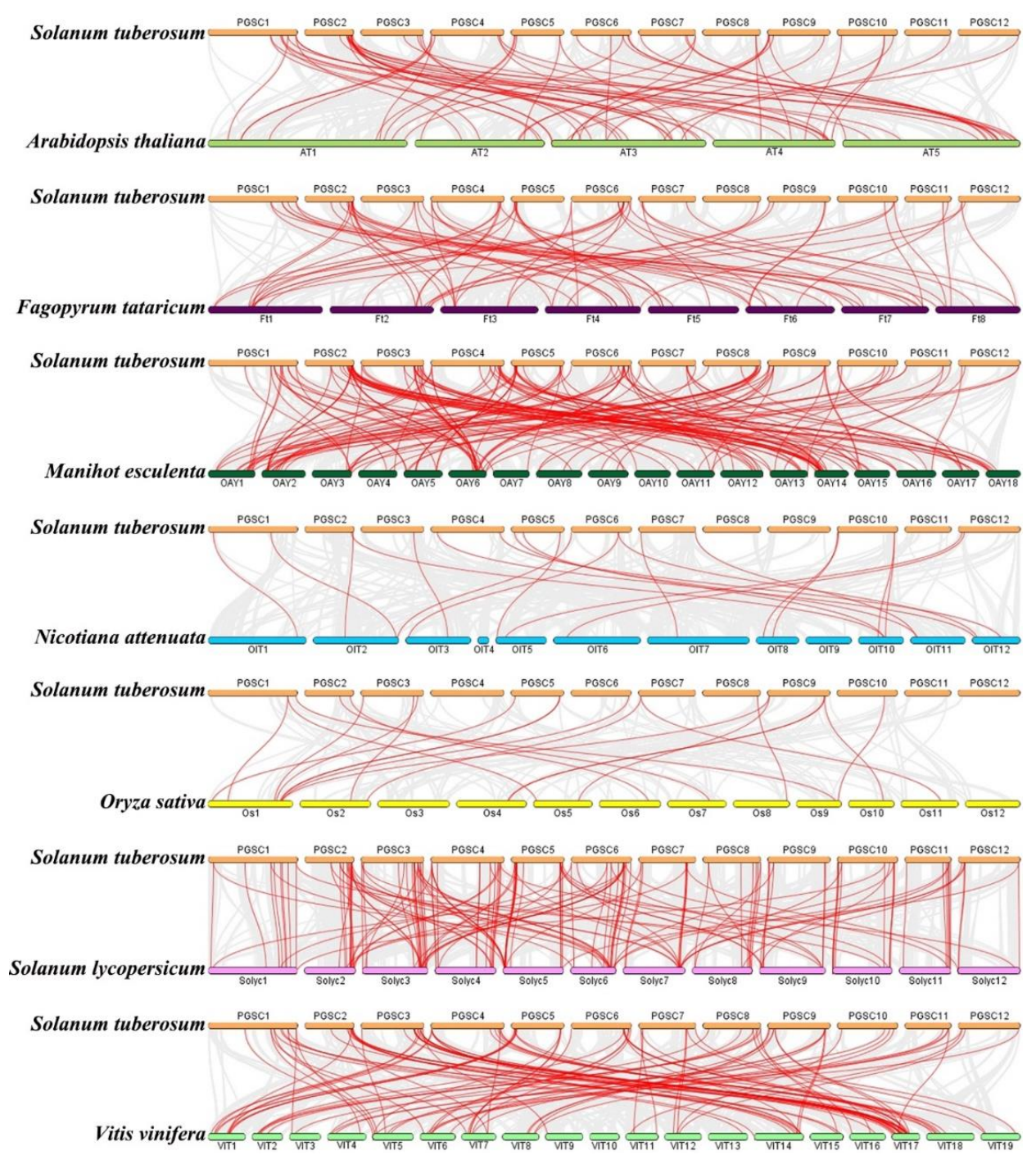

Figure 6. Synteny analyses between the $M Y B$ genes of potato and seven representative plant species. Gray lines in the background indicate collinear blocks within potato and other plant genomes, while red lines highlight syntenic MYB gene pairs. 


\subsection{Expression Patterns of StMYB Genes in Different Plant Tissues}

$M Y B$ genes in different subgroups may perform different functions during the growth and development of potato. To explore their specific functions, we selected a $S t M Y B$ gene from each subgroup and determined their expression levels in roots, stems, leaves, flowers, shoots, stolons, young tubers, and mature tubers by quantitative real-time polymerase chain reaction (qRT-PCR) (Figure 7A). With the exception of StMYB59, StMYB127, StMYB16, and StMYB141, other genes were expressed in all tissues, but the expression patterns of each gene were different. Except for the high expression of StMYB59 in mature tubers, the expression of $S t M Y B 59$ was very low in other tissues, but not in stems or flowers. StMYB127 was only expressed in the root, and the expression level was low. StMYB16 was not expressed in stems. However, StMYB141 was not expressed in leaves, and its expression level was low in stems. The expression levels of StMYB60, StMYB44, StMYB16, StMYB141, and StMYB127 were the highest in roots, among which $S t M Y B 127$ was a root-specific gene. StMYB12, StMYB6, StMYB133, StMYB74, StMYB1, StMYB75, and StMYB89 were most expressed in flowers. StMYB59 and StMYB53 were most expressed in tubers. StMYB3, StMYB27, and StMYB19 were most expressed in stolons. StMYB119 and StMYB143 were most expressed in shoots. StMYB11 and StMYB116 were most expressed in stems (Figure 7A).

We analyzed the correlation between the expression of these genes in different tissues. It can be seen from Figure $7 \mathrm{~B}$ that the expression levels of more than half of the genes in different tissues were positively correlated, among which StMYB3 and StMYB44, as well as StMYB12 and StMYB133, were significantly positively correlated.

\subsection{Expression Analysis of StMYB Genes under Phytohormone and Abiotic Stresses}

The expression levels of $S t M Y B$ genes in ABA, IAA, GA3, high temperature $\left(35^{\circ} \mathrm{C}\right)$, drought, and $\mathrm{NaCl}$ were measured by qRT-PCR (Figure 8A). As can be seen from Figure 8A, there were significant expression changes of these genes under different treatments. Except that StMYB127 and StMYB141 did not respond to hormone or stress treatments, all genes responded to hormone and abiotic stress treatments, but each gene had different expression patterns under different treatments. The expression levels of StMYB6 decreased under all stress treatments, StMYB27 and StMYB116 decreased under high temperature treatment, StMYB133 decreased under drought and high-temperature treatment, and the expression levels of other genes increased under hormone and stress treatments. StMYB60, StMYB3, StMYB44, StMYB16, and StMYB19 had the highest expression levels under ABA treatment; StMYB12, StMYB59, StMYB133, StMYB27, StMYB11, and StMYB143 had the highest expression levels under GA treatment; StMYB119 and StMYB116 had the highest expression levels under drought stress; StMYB74 had the highest expression level under salt stress; StMYB1, StMYB75, and StMYB53 had the highest expression levels under high-temperature treatment; and only StMYB89 had the highest expression level under IAA treatment. 
A
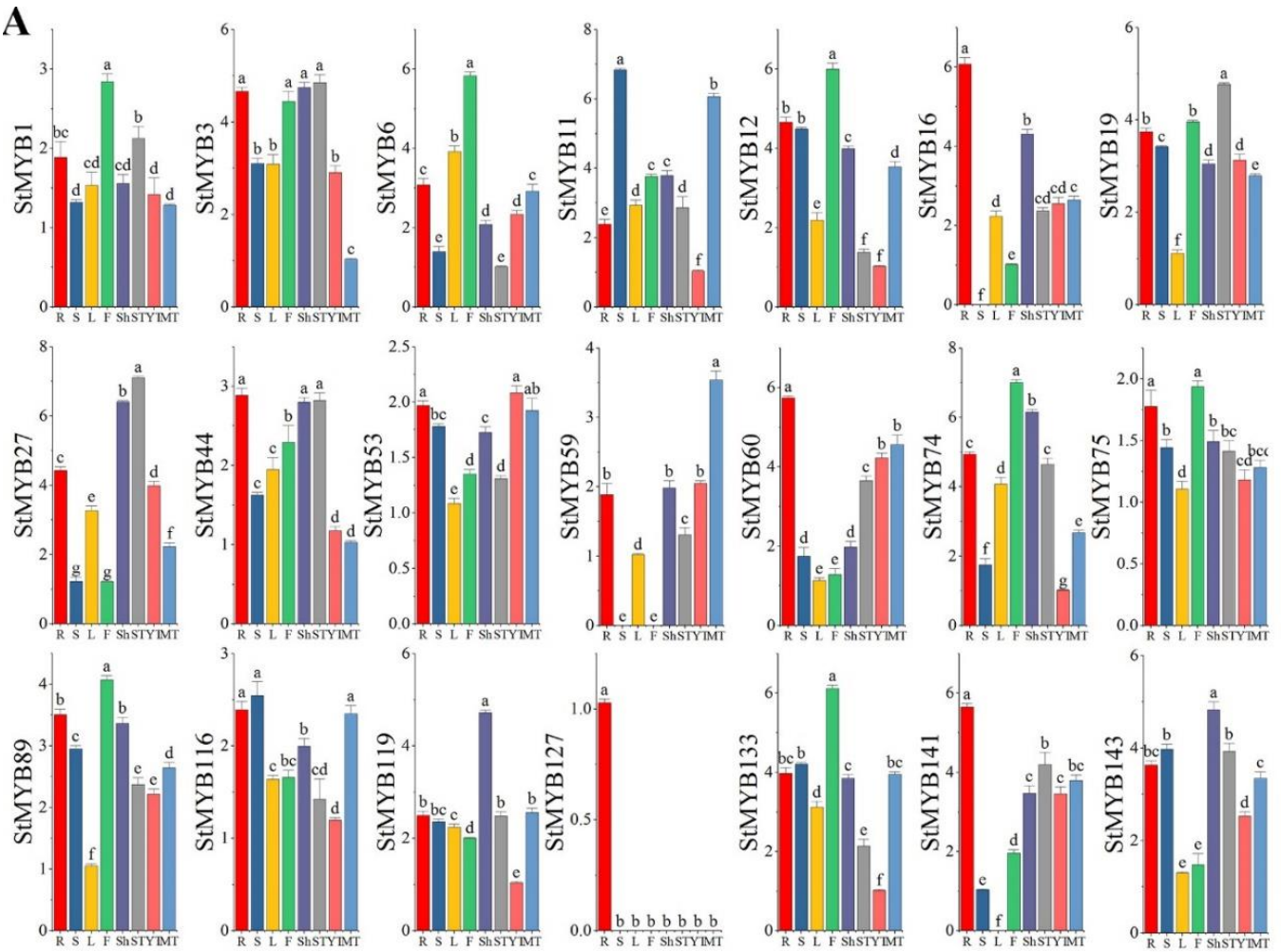

B

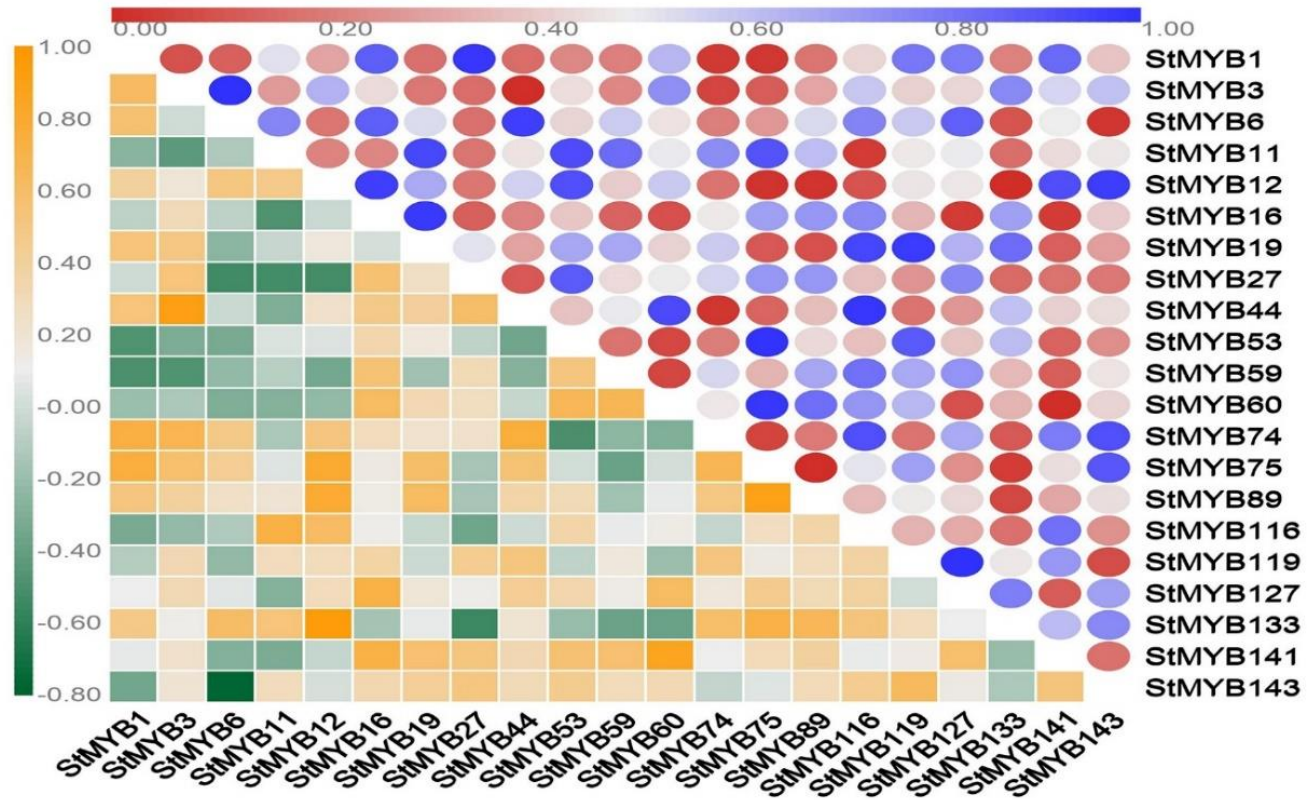

Figure 7. Tissue-specific gene expression of 21 potato $M Y B$ genes and the correlation between the gene expression patterns of StMYBs. (A) The expression patterns of 21 potato MYB genes in roots, stems, leave, flowers, shoots, stolons, young tuber, and mature tuber were examined by quantitative real-time polymerase chain reaction (qRT-PCR). The expression pattern of each gene was calculated based on the minimum expression of each gene in different tissues. Error bars were obtained from three measurements. Lowercase letter(s) above the bars indicate significant differences $(\alpha=0.05$, least significant difference (LSD)) among the treatments. (B) Different colors represent the correlation of $S t M Y B$ gene expression patterns in different tissues. Different colors in the lower left corner represent the correlation of $S t M Y B$ gene expression patterns in different tissues, while the different colors in the upper right corner represent the $p$-value of the correlation. 
A
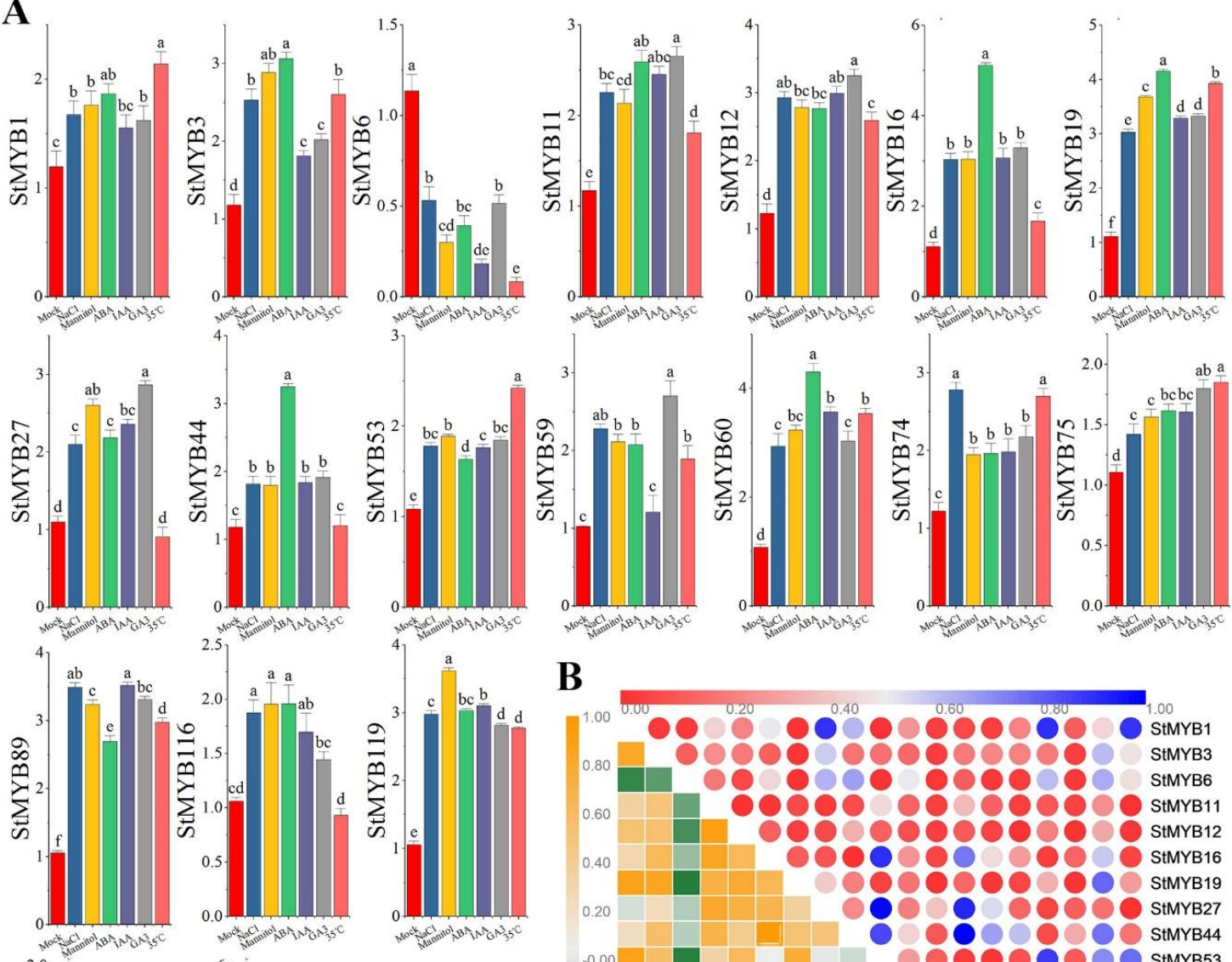

B
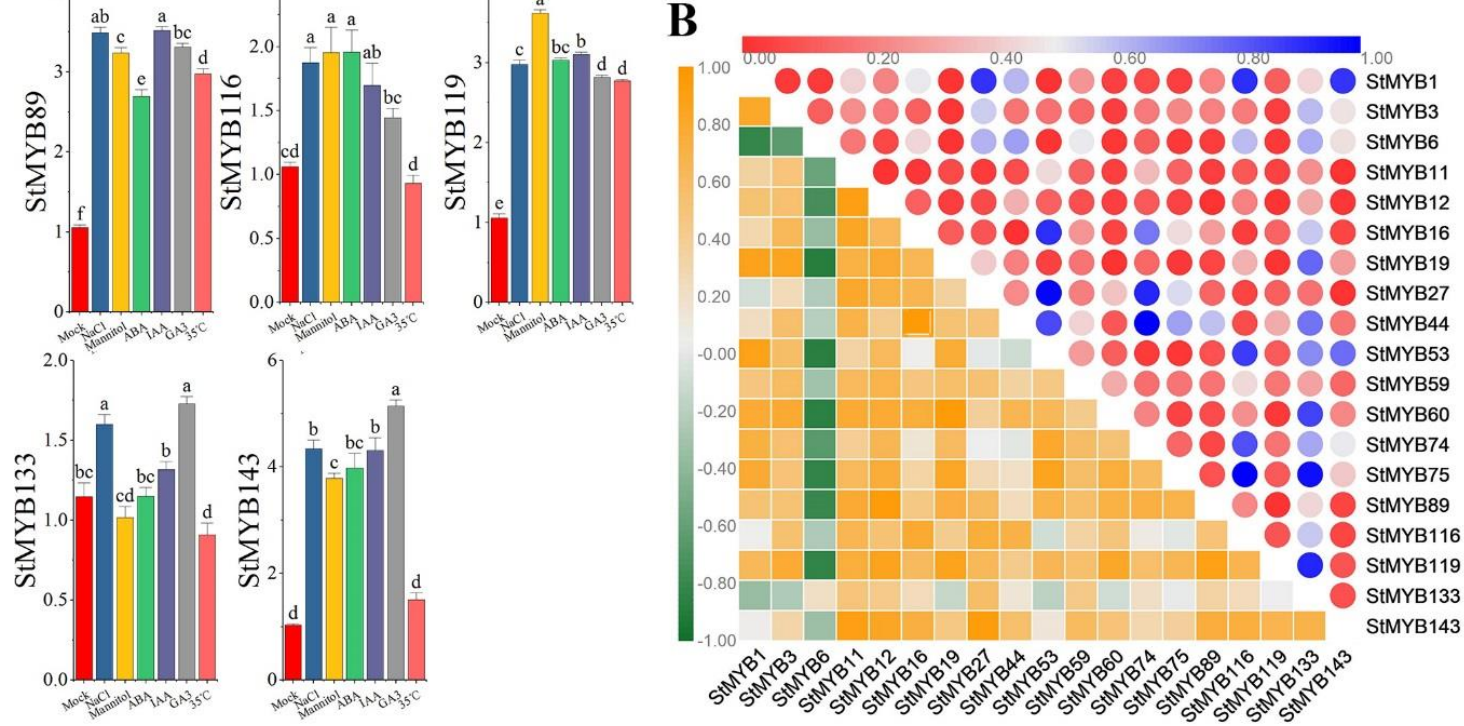

Figure 8. Gene expression of 19 potato $M Y B$ genes under hormones and abiotic stress and the correlation between the gene expression patterns of $S t M Y B$ genes. (A) The expression patterns of 19 potato $M Y B$ genes under hormones and abiotic stress were examined using a qRT-PCR assay. Error bars were obtained from three measurements. Lowercase letter(s) above the bars indicate significant differences ( $\alpha=0.05$, LSD) among the treatments. (B) Different colors represent the correlation of StMYB gene expression patterns under different hormone and abiotic stress treatments. Different colors in the lower left corner represent the correlation of $S t M Y B$ gene expression patterns under different hormone and abiotic stress treatments, while the different colors in the upper right corner represent the $p$-value of the correlation.

We analyzed the correlations between the expression levels of these genes under hormone and abiotic stresses. As can be seen from Figure 8B, the expression levels of most genes under hormone and abiotic stresses were positively correlated, among which StMYB1 was significantly positively correlated with the expression levels of StMYB75, StMYB60, StMYB53, StMYB19, StMYB6, and StMYB3.

\subsection{Subcellular Localization of StMYB6 and StMYB19}

To explore the subcellular localization of StMYB proteins, the representative proteins StMYB6, StMYB19, and enhanced green fluorescent protein (eGFP) were constructed in the CAMV35S promoter, 
and then transferred into the Arabidopsis thaliana (L.) Heynh. protoplasts. The CAMV35S driven eGFP was transferred into Arabidopsis thaliana (L.) Heynh. protoplasts as a control (Figure 9). It is found that eGFP protein was distributed in different subcellular locations (Figure 9). However, the fusion proteins of StMYB6 with eGFP and StMYB19 with eGFP were mainly distributed in the nucleus (Figure 9).

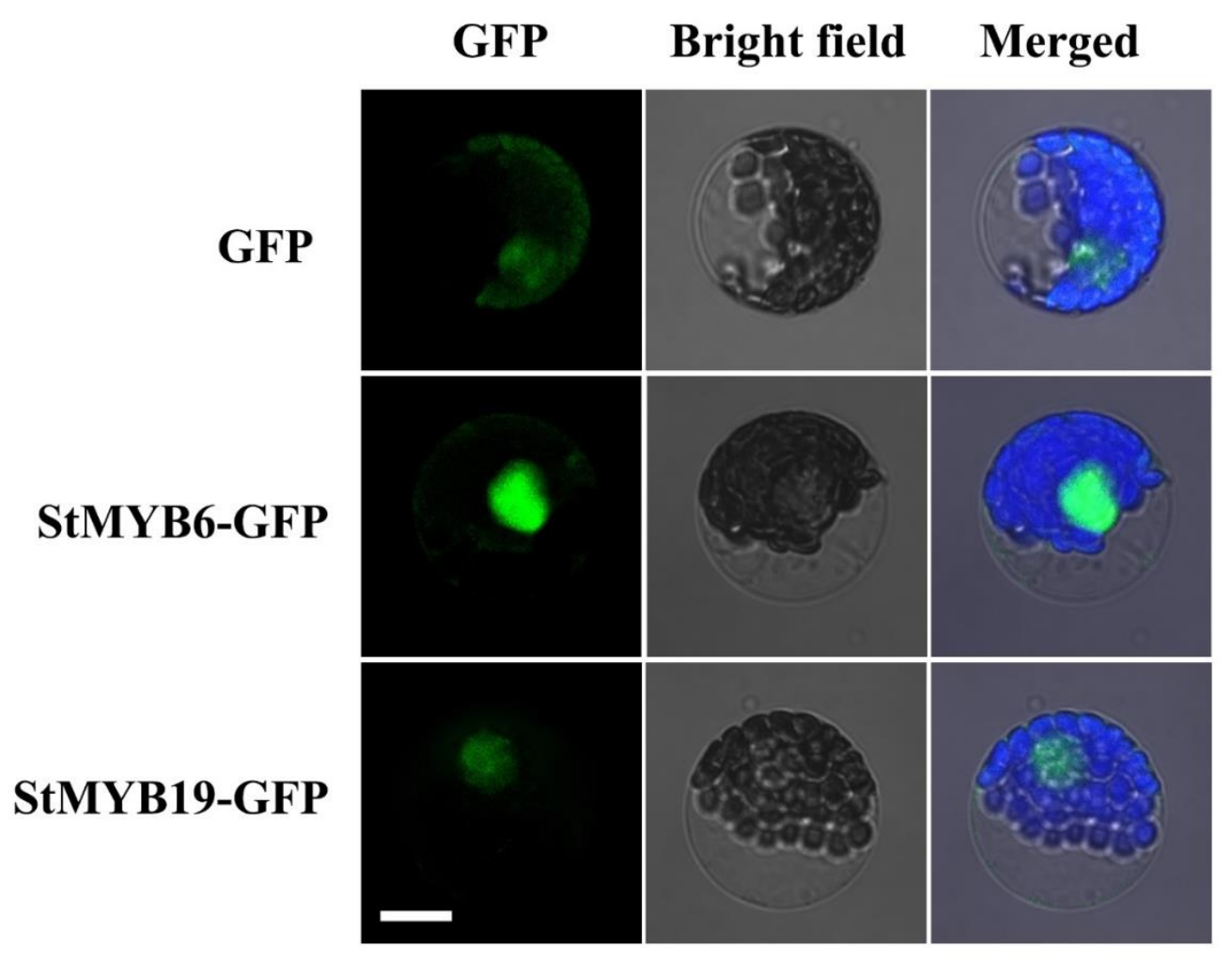

Figure 9. Subcellular localization of StMYB6 and StMYB19 in Arabidopsis thaliana (L.) Heynh. protoplasts. Enhanced green fluorescent protein (eGFP), StMYB6-eGFP, and StMYB19-eGFP under the control of the CaMV35S promoter separately transiently expressed in Arabidopsis thaliana (L.) Heynh. protoplasts. eGFP fluorescence signal was observed by Olympus confocal microscope. Scale bars $=10 \mu \mathrm{m}$.

\section{Discussion}

\subsection{Evolutionary Analysis of the StMYB Gene Family}

The MYB gene family is one of the largest transcription factor families in plants [5]. MYB genes play key roles in plant growth and development and a large number of biological processes [31-34]. With the continuous publication of genome data, the $M Y B$ gene family has been identified in many plants at the whole-genome level, including Manihot esculenta Crantz (299 members) [35], Solanum lycopersicum (121 members) [36], Brassica napus L. (249 members) [37], Oryza sativa L. (155 members) [23], Arabidopsis thaliana (L.) Heynh. (197 members) [23], soybean (244 members) [38], and so on. The genome size of Manihot esculenta Crantz is 742 MB [39], the Solanum lycopersicum genome size is 828.349 MB [40], the Brassica napus L. genome size is $912.169 \mathrm{MB}$ [41], the rice genome size is $466 \mathrm{Mb}$ [42], the Arabidopsis thaliana (L.) Heynh. genome size is $125 \mathrm{MB}$ [43], and the soybean genome size is 1.025 GB [44]. The number of $M Y B$ genes identified in these plants is different, and their genome size is also different. By comparing the genome size of these plants and the number of identified $M Y B$ genes, it is found that the number of $M Y B$ genes in each plant did not depend entirely on the genome size. For example, the genome size of Solanum lycopersicum was medium, but the number of MYB genes in Solanum lycopersicum was the lowest.

Gene duplication events are the main reason for the expansion and evolution of plant genomes, and they are also the main factors promoting the expansion of gene families. The mechanisms of 
unequal crossing-over, duplication-dependent chromosome breakage, and nonhomologous exchange all lead to duplication events. Li et al. identified 8 pairs of tandem-duplicated StMYB genes and 31 pairs of segmental-duplicated $S t M Y B$ genes from $230 S t M Y B$ genes [30]. In this study, 36 pairs of tandem-duplicated $S t M Y B$ and 41 pairs of segmental-duplicated StMYB genes were identified from 158 StMYB genes (Figure 3). The different number of tandem duplication gene pairs we identified was because of the different identification criteria. We defined adjacent genes within $200 \mathrm{~kb}$ as tandem duplication gene pairs [45], while Li et al. defined adjacent genes with more than $70 \%$ identity in $200 \mathrm{~kb}$ as tandem duplication genes [30]. Similarly, apple, pear, and grape also experienced duplication events that led to the expansion of the MYB gene family in their genomes, suggesting that tandem duplication and segmental duplication together have promoted the expansion of the MYB gene family in different plants during the evolutionary process $[13,24,46,47]$. Duplicated genes may undergo different selection processes: achieving non-functionalization through silencing, achieving neofunctionalization by acquiring new functions, or being subfunctionalized by dividing the original functions of ancestral genes [48-50]. In this study, the expression levels of the identified tandem-duplicated genes were different in tissues (Figure 7). For example, StMYB3 and StMYB4 were a pair of tandem-duplicated genes, StMYB4 was not expressed, but StMYB3 was expressed in different tissues (Figure 3, Figure 7). We speculate that the reason may be that $S T M Y B 4$ lost the function of the original genes during evolution.

To further explain the evolutionary relationship of $M Y B$ genes in potato, we constructed a phylogenetic tree including potato, Arabidopsis thaliana (L.) Heynh., Manihot esculenta Crantz, Solanum lycopersicum, Vitis vinifera L., and Oryza sativa L. At the same time, we analyzed the motif compositions of $M Y B$ genes in these plants (Figure 5). From Figure 5, we can see that most of the MYB genes in all plants contained motif 1 , motif 2 , motif 4 , and motif 5 ; although the motif composition of each subgroup was different, the motif composition within the same subgroup was similar. The motif compositions of the closely related members of the phylogenetic tree were consistent, which was also reported for Phyllostachys edulis, indicating that the MYB family is highly conserved between different plants [51]. Distinguished with Li et al.'s research [30], we analyzed the motif composition of MYB genes in different species, which can explore the conservativeness of $M Y B$ genes in different species and lay a foundation for syntenic analysis. By comparing the syntenic analysis of MYB genes in potato and other plants, we found that $S t M Y B$ genes had the most homologous gene pairs with $M Y B$ genes in Solanum lycopersicum and Manihot esculenta Crantz, followed by Vitis vinifera L, which was consistent with Li et al. [30]. These results suggest that MYB genes in different plants may come from a common ancestor.

\subsection{StMYB Genes Play Crucial Roles in Potato Growth and Development and Response to Phytohormone and Abiotic Stresses}

The high-temperature, drought, and abiotic stresses seriously affected the yield of potato [52,53]. Secondary metabolites in plants, including flavonoids and anthocyanins, can help plants adapt to changing environments. Therefore, it is necessary to improve the resistance of the potato by regulating the genes related to the secondary metabolites, thereby increasing the yield. MYB genes can regulate the development of trichomes and root hairs [54,55], and they play a vital role in regulating the synthesis of plant secondary metabolites, such as flavonoids and anthocyanins [17,56-58], as well as helping plants resist abiotic stress [59-61]. To screen the StMYB genes that potentially regulate growth, development, and secondary metabolic synthesis, this study selected genes from each subgroup and measured their expression levels in different tissues. Different from our study, Li et al. focused on the expression levels of genes in SGs 14 and 20 in different tissues [30]. AtMYB11, AtMYB12, and AtMYB111 were members of MYB SG 7 in Arabidopsis thaliana (L.) Heynh., and regulate flavonoid biosynthesis in all tissues [17]. StMYB6 also belonged to SG 7, which is a homologous gene of AtMYB11, AtMYB12, and AtMYB111. qRT-PCR showed that it was expressed in all tissues of potato, but the highest expression level was found in flowers (Figure 7). In the future, we can verify whether StMYB6 can regulate flavonoid synthesis through more in-depth experiments. AtMYB genes from SG 4 are 
transcriptional repressors of different branches of the phenylalanine metabolic pathway $[62,63]$. It has been reported that $A t M Y B 4$ negatively regulates the formation of sinapate ester in the absence of ultraviolet radiation. When exposed to UV-B, the expression level of AtMYB4 decreases, which increases the content of sinapate ester and plays a role in ultraviolet protection [62]. AtMYB7 negatively regulates the synthesis of flavonoids and is a newly discovered transcriptional inhibitor involved in ultraviolet protection [64]. StMYB89 and StMYB12 also belonged to SG 4, and they were expressed in all tissues of potato, but were highest in mature flowers (Figure 1, Figure 4). Under phytohormone and abiotic stresses, their expression levels were also very high (Figure 8). StMYB12 was the most responsive to GA, while StMYB89 was the most responsive to IAA (Figure 8). StMYB89 and StMYB12 may play a crucial role in the growth and development of potato and resistance to adverse external environments. They can be used as candidate genes to improve potato quality, and their specific functions will be determined in future studies.

The expression of $M Y B$ genes is mainly regulated by a variety of hormones and abiotic stresses, including ABA, gibberellin, salt, drought, and high temperature. AtMYB60 and AtMYB96 from SG 1 can regulate stomatal movement and participate in drought stress through the ABA signaling pathway [65-67]. AtMYB33 and AtMYB101 from SG 18 and AtMYB13 and AtMYB15 from SG 2 respond to ABA expression [68,69]. AtMYB2 from SG 20 is induced by ABA to regulate salt stress, and AtMYB108 is involved in biotic and abiotic stresses [59,70]. AtMYB44 from SG 22 regulates ABA-mediated stomatal closure under abiotic stress, and AtMYB70, AtMYB73, and AtMYB77 in this SG are also involved in stress responses [71]. In order to explore StMYB genes involved in hormonal and abiotic stresses, we focused on the expression levels of StMYB genes from SG 1, SG 2, SG 18, SG 20, and SG 22 under different stress treatments, while Li et al. only focused on the expression patterns of genes of SG 1, SG 2, and SG 22 under stresses [30]. Using qRT-PCR, we found that StMYB44 of SG 1, StMYB27 of SG 2, StMYB1 of SG 18, StMYB3 and StMYB53 of SG 20, and StMYB19 of SG 22, which were homologous to stress responses genes, were expressed in all tissues. This indicated that they may play important roles in the potato growth and development. We predicted cis-acting elements in these genes promoters and found that their promoter regions contained multiple phytohormone responsiveness elements, and some StMYB genes contained the same copies of cis-acting elements, which may enhance the transcriptional regulation of genes and enable plants to adapt to changes in the environment (Figure 2D). To further identify candidate genes involved in hormone signaling pathways or abiotic stress responses, we measured their expression levels under hormone, salt, drought, and high-temperature treatments. The results showed that these genes were expressed under hormone and abiotic stresses, but the expression pattern of each gene was different (Figure 8). StMYB44 had the highest expression under ABA treatment, which was consistent with the prediction of the composition of cis-acting elements in its promoter (Figure 2D, Figure 8). We can verify whether StMYB44 performs the same functions as $A t M Y B 60$ and AtMYB96 in the future $[65,66]$. Except for the low expression level of StMYB27 under high-temperature treatment, the expression level of StMYB27 was high under all treatments, especially under the treatment of GA, which was consistent with the prediction of its promoter cis-acting elements (Figure 2D, Figure 8). StMYB3, StMYB1, and StMYB53 were expressed under different treatments; StMYB3 was the most responsive to ABA treatment, and StMYB1 and $S t M Y B 53$ were the most responsive to high-temperature treatment (Figure 8). Their specific functions need to be further verified in the future. StMYB19 was a gene that responded to hormone and abiotic stresses with high expression, which is consistent with the cis-acting element in its promoter (Figure 2D, Figure 8). We speculate that StMYB19 may also be a gene that performs functions in the abiotic stress response, but the mechanism of its executive function is still unclear, which needs to be further verified in future experiments. Some $S t M Y B$ genes screened in this study were potentially involved in ABA, GA, high temperature, and other abiotic stresses, while some StMYB genes screened by Li et al. were involved in salt tolerance [30].

In conclusion, a total of 158 StMYB genes were identified from potato genome. The gene structure, motif composition, chromosome localization, evolutionary relationship, expression patterns in different 
tissues, and response to hormone and abiotic stresses of $S t M Y B$ genes were comprehensively analyzed. We have identified several potential candidate genes that regulate the synthesis of potato flavonoids or participate in hormones and stress responses. Our research lays a foundation for the future investigation of the potential functions of $S t M Y B$ genes in the growth and development of potato.

\section{Materials and Methods}

\subsection{Plant Materials and Growth Conditions}

Potato Cultivar chuanyu 117 were used in this study. The potato plants were grown in a greenhouse at the College of Life Sciences, Sichuan Agricultural University, China. Different plant tissues were collected at different times after potato germination. Young buds were collected two weeks after germination. Roots, stems, leaves, and flowers were collected at the flowering stage. Stolons and young tubers were collected 15 days after anthesis. Mature tubers were collected approximately three months after potato germination. All samples were collected from three plants with similar growth. After the samples were collected, they were immediately put in liquid nitrogen and then put at $-80^{\circ} \mathrm{C}$ for storage.

The potatoes used for hormone and abiotic stresses were grown in MS medium with a $\mathrm{pH}$ of $5.8,3 \%$ sucrose, and $1.5 \%$ agar in an external environment of $23{ }^{\circ} \mathrm{C}$, with a photoperiod of $16 \mathrm{~h}$ of light and $8 \mathrm{~h}$ of darkness. Potato plantlets at four weeks were subjected to hormone, heat, drought, and salt stresses. For hormone treatment, potato plantlets were treated with $50 \mu \mathrm{M}$ ABA, $10 \mu \mathrm{M}$ $\mathrm{IAA}$, and $50 \mu \mathrm{M} \mathrm{GA} 3$ for $24 \mathrm{~h}$. For temperature stress, plantlets were exposed to $35^{\circ} \mathrm{C}$. For drought treatment, plantlets were treated with $260 \mu \mathrm{M}$ mannitol for $24 \mathrm{~h}$. For salt treatment, potato plantlets were treated with $150 \mathrm{mM} \mathrm{NaCl}$ for $24 \mathrm{~h}$. Three plants with similar growth were selected for each stress treatment. After $24 \mathrm{~h}$ of different stress treatments, the third leaf from the top of the plant was collected. The collected samples were stored at $-80^{\circ} \mathrm{C}$.

\subsection{Identification of the StMYB Genes in Potato}

We downloaded the potato genome sequence from the Potato Genome Sequencing Consortium (PGSC, http://potato.plantbiology.msu.edu/). We obtained the hidden Markov model (HMM) profile of the MYB domain (PF00249) through the Pfam protein family database (http://pfam.sanger.ac.uk/). The specific identification method of $M Y B$ genes was as follows. We downloaded the amino acid sequences of all MYB genes in Arabidopsis thaliana (L.) Heynh. from the Arabidopsis Information Resource (TAIR) library. All $M Y B$ genes were identified from the potato genome using downloaded $A t M Y B$ gene sequences as target sequences. The conserved domains of the potato MYB genes identified above were analyzed, and the genes that did not contain the PF00249 conserved domain were removed. Then, the identified potato $M Y B$ genes were run against BLASTp at National Center for Biotechnology Information (NCBI) to determine whether they belonged to the MYB gene family. Finally, we identified 158 StMYB genes from the potato genome. Basic information about these genes, including PIs, MWs, and subcellular localization, was predicted through the ExPASy website (https://web.expasy.org/protparam/).

\subsection{Phylogenetics, Intron-Exon Structure, Motif Composition, and Cis-Acting Elements}

We downloaded MYB protein sequences (Arabidopsis thaliana (L.) Heynh., Solanum lycopersicum, Vitis vinifera L., Manihot esculenta Crantz, and Oryza sativa L.) from the UniProt database (https: //www.uniprot.org/) for constructing a multispecies maximum likelihood (ML) phylogenetic tree. Multiple amino acid sequences of MYB proteins from different plants were aligned using MUSCLE [72]. The ML phylogenetic tree including potato, Arabidopsis thaliana (L.) Heynh., Solanum lycopersicum, Vitis vinifera L., Manihot esculenta Crantz, and Oryza sativa L. was constructed with Mega 7.0 [73], and the specific parameters were the JTT $+\mathrm{G}+\mathrm{F}$ model and 1000 bootstrap replications. The ML phylogenetic tree of potato and Arabidopsis thaliana (L.) Heynh. was constructed by the same method mentioned 
above, and the MYB protein sequences of potato and Arabidopsis thaliana (L.) Heynh. were aligned using MUSCLE before constructing the phylogenetic tree. The DNA and cDNA sequences of MYB genes were compared by online Gene Structure Display Service (http://gsds.cbi.pku.edu.cn/) to predict the structure of introns and exons. The conserved motifs of MYB proteins were determined by the Multiple Em for Motif Elicitation (MEME) online program (http://meme-suite.org/tools/meme) (Table S2). PlantCARE software (http://bioinformatics.psb.ugent.be/webtools/plantcare/html/?tdsourcetag=s_pcqq_aiomsg) was used to predict the cis-acting elements within $2000 \mathrm{bp}$ upstream of all StMYB genes.

\subsection{Chromosomal Mapping, Gene Duplication, and Synteny with Other Plants}

We obtained StMYB gene localization information from circos and observed that all StMYB genes were located on different chromosomes of potato [74]. StMYB gene duplication events were analyzed with a multiple collinear scanning toolkit (MCScanX) [75]. The syntenic relationship between the StMYB genes and MYB genes from Solanum lycopersicum, Manihot esculenta Crantz, Vitis vinifera L., Fagopyrum tataricum Gaertn., Arabidopsis thaliana (L.) Heynh., Nicotiana attenuate, and Oryza sativa L. were determined using Dual Synteny Plotter software [76].

\subsection{Expression Analysis of the StMYB Genes by qRT-PCR}

Total RNA was extracted from the samples using the RNAout Kit (TIANGEN, China), and cDNA was synthesized using the PrimeScript ${ }^{\mathrm{TM}} 1$ st Strand cDNA Synthesis Kit (Takara, Japan) according to the manufacturer's instructions. The expression levels of StMYB genes identified in the roots, stems, leaves, flowers, shoots, stolons, young tubers, and mature tubers were measured by qRT-PCR. Meanwhile, the expression levels of $S t M Y B$ genes in potatoes treated with ABA, IAA, GA3, NaCl, mannitol, and heat were also measured. The qRT-PCR primers of the StMYB genes listed in Table S4 were obtained by online software primer 3 (https://www.ncbi.nlm.nih.gov/tools/primer-blast/). The qRT-PCR used elongation factor $1-\alpha($ ef $1 \alpha)$ as internal reference gene [77]. The correlative expression data were calculated based on the $2^{-\Delta \Delta C T}$ method [78]. Three biological replicates were performed in the qRT-PCR experiment, and three technical replicates were performed in each biological replicate.

\subsection{Subcellular Localization}

The coding regions of StMYB6 and StMYB19 were amplified by PCR and fused to the pCAMBIA2300-eGFP plant expression vector. Arabidopsis thaliana (L.) Heynh. protoplasts were prepared from leaves of Arabidopsis thaliana (L.) Heynh. eGFP, StMYB6-eGFP, and StMYB19-eGFP were transferred into Arabidopsis thaliana (L.) Heynh. protoplasts. After $12 \mathrm{~h}$ transformation, the eGFP fluorescence was observed under an Olympus confocal microscope.

\subsection{Statistical Analysis}

We processed and analyzed our experimental data with the Origin Pro 2018b (OriginLab Corporation, Northampton, Massachusetts, USA), and we used the least significant difference (LSD) test to compare the data.

Supplementary Materials: Supplementary materials can be found at http://www.mdpi.com/1422-0067/20/19/ $4847 / s 1$.

Author Contributions: M.L. planned and designed the research and analyzed data. W.S. wrote the original manuscript. W.S. and Z.M. determined the expression of genes by qRT-PCR, identified StMYB family genes and visualized their structures. M.L. and H.C. performed the evolutionary analysis of StMYB genes and several different species, performed $S t M Y B$ genes chromosome distribution, gene duplication and synchronous analysis. M.L. reviewed and edited the manuscript. H.C. supervised the research. W.S. and Z.M. contributed equally. All authors read and approved the final manuscript.

Funding: This research received no external funding. 
Acknowledgments: We thank all the colleagues in our laboratory for providing useful discussions and technical assistance. We are very grateful to the editor and reviewers for critically evaluating the manuscript and providing constructive comments for its improvement.

Conflicts of Interest: The authors declare no conflict of interest.

\section{Abbreviations}

$\begin{array}{ll}\text { ABA } & \text { abscisic acid } \\ \text { GA3 } & \text { gibberellin acid 3 } \\ \text { GFP } & \text { green fluorescent protein } \\ \text { HTH } & \text { helix-turn-helix } \\ \text { IAA } & \text { indole-3-acetic acid } \\ \text { MEME } & \text { Multiple Em for Motif Elicitation } \\ \text { MWs } & \text { molecular weights } \\ \text { NCBI } & \text { National Center for Biotechnology Information } \\ \text { PIs } & \text { isoelectric points } \\ \text { qRT-PCR } & \text { quantitative real-time polymerase chain reaction } \\ \text { R } & \text { repeat } \\ \text { SA } & \text { salicylic acid } \\ \text { SGs } & \text { subgroups } \\ \text { StMYB } & \text { Solanum tuberosum L. MYB } \\ \text { TFs } & \text { transcription factors }\end{array}$

\section{References}

1. Goode, J.A.; Organizers, D.C. Improving Plant Drought, Salt and Freezing Tolerance by Gene Transfer of a Single Stress-Inducible Transcription Factor. Nat. Biotechnol. 2007, 17, 287-291.

2. Valliyodan, B.; Nguyen, H.T. Understanding regulatory networks and engineering for enhanced drought tolerance in plants. Curr. Opin. Plant Biol. 2006, 9, 189-195. [CrossRef]

3. Wang, Z.; Tang, J.; Hu, R.; Wu, P.; Hou, X.L.; Song, X.M.; Xiong, A.S. Genome-wide analysis of the R2R3-MYB transcription factor genes in Chinese cabbage (Brassica rapa ssp. pekinensis) reveals their stress and hormone responsive patterns. BMC Genom. 2015, 16, 17-38. [CrossRef]

4. Li, C.; Ng, K.Y.; Fan, L.M. MYB transcription factors, active players in abiotic stress signaling. Environ. Exp. Bot. 2015, 114, 80-91. [CrossRef]

5. Dubos, C.; Stracke, R.; Grotewold, E.; Weisshaar, B.; Martin, C.; Lepiniec, L. MYB transcription factors in Arabidopsis. Trends Plant Sci. 2010, 15, 573-581. [CrossRef] [PubMed]

6. Li, Q.; Zhang, C.; Li, J.; Wang, L.; Ren, Z. Genome-wide identification and characterization of R2R3MYB family in Cucumis sativus. PLoS ONE 2012, 7, e47576. [CrossRef]

7. Nozomi, H.; Kiichi, K.; Masatake, M.; Satoshi, A.; Minoru, K.; Taku, D.; Kaoru, S.; Isabel, M.; Ute, V.; Gerd, J. R1R2R3-Myb proteins positively regulate cytokinesis through activation of KNOLLE transcription in Arabidopsis thaliana. Development 2007, 134, 1101-1110.

8. Stracke, R.; Werber, M.; Weisshaar, B. The R2R3-MYB gene family in Arabidopsis thaliana. Curr. Opin. Plant Biol. 2001, 4, 447-456. [CrossRef]

9. Chen, Y.; Yang, X.; He, K.; Liu, M.; Li, J.; Gao, Z.; Lin, Z.; Zhang, Y.; Wang, X.; Qiu, X. The MYB Transcription Factor Superfamily of Arabidopsis: Expression Analysis and Phylogenetic Comparison with the Rice MYB Family. Plant Mol. Biol. 2006, 60, 107-124.

10. Jiao, D.; Ming, L.; Huang, L.; Mei, Y.; Yang, P. Genome-Wide Analysis of the R2R3 MYB Subfamily Genes in Lotus (Nelumbo nucifera). Plant Mol. Biol. Report. 2016, 34, 11.

11. Rosinski, J.A.; Atchley, W.R. Molecular Evolution of the Myb Family of Transcription Factors: Evidence for Polyphyletic Origin. J. Mol. Evol. 1998, 46, 74-83. [CrossRef]

12. Jiang, C.; Gu, J.; Chopra, S.; Gu, X.; Peterson, T. Ordered origin of the typical two- and three-repeat Myb genes. Gene 2004, 326, 13-22. [CrossRef] 
13. Matus, J.T.; Aquea, F.; Arce-Johnson, P. Analysis of the grape MYB R2R3 subfamily reveals expanded wine quality-related clades and conserved gene structure organization across Vitis and Arabidopsis genomes. BMC Plant Biol. 2008, 8, 83-98. [CrossRef]

14. Dias, A.P.; Braun, E.L.; Mcmullen, M.D.; Erich, G. Recently duplicated maize R2R3 Myb genes provide evidence for distinct mechanisms of evolutionary divergence after duplication. Plant Physiol. 2003, 131, 610-620. [CrossRef]

15. Martin, C.; Paz-Ares, J. MYB transcription factors in plants. Trends Genet. 1997, 13, 67-73. [CrossRef]

16. Jin, H.; Martin, C. Multifunctionality and diversity within the plant MYB-gene family. Plant Mol. Biol. 1999, 41, 577-585. [CrossRef]

17. Stracke, R.; Ishihara, H.G.; Barsch, A.; Mehrtens, F.; Niehaus, K.; Weisshaar, B. Differential regulation of closely related R2R3-MYB transcription factors controls flavonol accumulation in different parts of the Arabidopsis thaliana seedling. Plant J. 2010, 50, 660-677. [CrossRef]

18. Gonzalez, A.; Zhao, M.; Leavitt, J.M.; Lloyd, A.M. Regulation of the anthocyanin biosynthetic pathway by the TTG1/bHLH/Myb transcriptional complex in Arabidopsis seedlings. Plant J. 2010, 53, 814-827. [CrossRef]

19. Lepiniec, L.; Debeaujon, I.; Routaboul, J.-M.; Baudry, A.; Pourcel, L.; Nesi, N.; Caboche, M. Genetics and Biochemistry of Seed Flavonoids. Annu. Rev. Plant Biol. 2006, 57, 405-430.

20. Lee, T.G.; Jang, C.S.; Kim, J.Y.; Kim, D.S.; Park, J.H.; Kim, D.Y.; Seo, Y.W. A Myb transcription factor (TaMyb1) from wheat roots is expressed during hypoxia: Roles in response to the oxygen concentration in root environment and abiotic stresses. Physiol. Plant. 2010, 129, 375-385. [CrossRef]

21. Mao, X.; Jia, D.; Li, A.; Zhang, H.; Tian, S.; Zhang, X.; Jia, J.; Jing, R. Transgenic expression of TaMYB2A confers enhanced tolerance to multiple abiotic stresses in Arabidopsis. Funct. Integr. Genom. 2011, 11, 445-465. [CrossRef]

22. Qin, Y.; Wang, M.; Tian, Y.; He, W.; Han, L.; Xia, G. Over-expression of TaMYB33 encoding a novel wheat MYB transcription factor increases salt and drought tolerance in Arabidopsis. Mol. Biol. Rep. 2012, 39, 7183-7192. [CrossRef]

23. Katiyar, A.; Smita, S.; Lenka, S.K.; Rajwanshi, R.; Chinnusamy, V.; Bansal, K.C. Genome-wide classification and expression analysis of MYB transcription factor families in rice and Arabidopsis. BMC Genom. 2012, 13, 544-563. [CrossRef]

24. Feng, S.; Xu, Y.; Long, Y.; Sun, S.; Wang, D.; Chen, X. Genome-wide identification and characterization of R2R3-MYB transcription factors in pear. Sci. Hortic. 2015, 197, 176-182. [CrossRef]

25. Stracke, R.; Holtgräwe, D.; Schneider, J.; Pucker, B.; Sörensen, T.R.; Weisshaar, B. Genome-wide identification and characterisation of R2R3-MYB genes in sugar beet ( Beta vulgaris ). BMC Plant Biol. 2014, 14, $249-266$. [CrossRef]

26. Salih, H.; Gong, W.; He, S.; Sun, G.; Sun, J.; Du, X. Genome-wide characterization and expression analysis of MYB transcription factors in Gossypium hirsutum. BMC Genet. 2016, 17, 129-141. [CrossRef]

27. Hai, D.; Bo-Run, F.; Si-Si, Y.; Yu-Bi, H.; Yi-Xiong, T. The R2R3-MYB transcription factor gene family in maize. PLoS ONE 2012, 7, e37463-e37475.

28. Dongjin, S.; Seok-Jun, M.; Seyoun, H.; Beom-Gi, K.; Ryeol, P.S.; Seong-Kon, L.; Hye-Jin, Y.; Hye Eun, L.; Hawk-Bin, K.; Dongwon, B. Expression of StMYB1R-1, a novel potato single MYB-like domain transcription factor, increases drought tolerance. Plant Physiol. 2011, 155, 421-432.

29. Cheng, Y.J.; Kim, M.D.; Deng, X.P.; Kwak, S.S.; Chen, W. Enhanced Salt Stress Tolerance in Transgenic Potato Plants Expressing IbMYB1, a Sweet Potato Transcription Factor. J. Microbiol. Biotechnol. 2013, 23, 1737-1746. [CrossRef]

30. Li, X.; Guo, C.; Ahmad, S.; Wang, Q.; Yu, J.; Liu, C.; Guo, Y. Systematic Analysis of MYB Family Genes in Potato and Their Multiple Roles in Development and Stress Responses. Biomolecules 2019, 9, 317. [CrossRef]

31. Qin, Z.; Hao, R.; Xu, Z.; Yang, W.; Jia, W.; Cheng, T.; Pan, H.; Zhang, Q. Isolation and functional characterization of a R2R3-MYB regulator of Prunus mume anthocyanin biosynthetic pathway. Plant Cell Tissue Organ Cult. 2017, 131, 13.

32. Gao, F.; Zhou, J.; Deng, R.Y.; Zhao, H.X.; Li, C.L.; Chen, H.; Suzuki, T.; Park, S.U.; Wu, Q. Overexpression of a tartary buckwheat R2R3-MYB transcription factor gene, FtMYB9, enhances tolerance to drought and salt stresses in transgenic Arabidopsis. J. Plant Physiol. 2017, 214, 81-90. [CrossRef] 
33. Meng, D.; He, M.; Bai, Y.; Xu, H.; Dandekar, A.M.; Fei, Z.; Cheng, L. Decreased sorbitol synthesis leads to abnormal stamen development and reduced pollen tube growth via an MYB transcription factor, MdMYB39L, in apple (Malus domestica). New Phytol. 2017, 217, 641-656. [CrossRef] 
34. Fang, Q.; Jiang, T.; Xu, L.; Liu, H.; Mao, H.; Wang, X.; Jiao, B.; Duan, Y.; Wang, Q.; Dong, Q. A salt-stress-regulator from the Poplar R2R3 MYB family integrates the regulation of lateral root emergence and ABA signaling to mediate salt stress tolerance in Arabidopsis. Plant Physiol. Biochem. 2017, 114, 100-110. [CrossRef]

35. Ruan, M.B.; Guo, X.; Wang, B.; Yang, Y.L.; Li, W.Q.; Yu, X.L.; Zhang, P.; Peng, M. Genome-wide characterization and expression analysis enables identification of abiotic stress-responsive MYB transcription factors in cassava (Manihot esculenta). J. Exp. Bot. 2017, 68, 3657-3672. [CrossRef]

36. Zhao, P.; Li, Q.; Li, J.; Wang, L.; Ren, Z. Genome-wide identification and characterization of R2R3MYB family in Solanum lycopersicum. Mol. Genet. Genom. 2014, 289, 1183-1207. [CrossRef]

37. Hajiebrahimi, A.; Owji, H.; Hemmati, S. Genome-wide identification, functional prediction and evolutionary analysis of R2R3-MYB superfamily in Brassica napus. Genome 2017, 60, 797. [CrossRef]

38. Du, H.; Yang, S.S.; Liang, Z.; Feng, B.R.; Liu, L.; Huang, Y.B.; Tang, Y.X. Genome-wide analysis of the MYB transcription factor superfamily in soybean. BMC Plant Biol. 2012, 12, 106-128. [CrossRef]

39. Wang, W.; Feng, B.; Xiao, J.; Xia, Z.; Zhou, X.; Li, P.; Zhang, W.; Wang, Y.; Møller, B.L.; Zhang, P. Cassava genome from a wild ancestor to cultivated varieties. Nat. Commun. 2014, 5, 5110-5119. [CrossRef]

40. Aoki, K.; Yano, K.; Suzuki, A.; Kawamura, S.; Sakurai, N.; Suda, K.; Kurabayashi, A.; Suzuki, T.; Tsugane, T.; Watanabe, M. Large-scale analysis of full-length cDNAs from the tomato ( Solanum lycopersicum ) cultivar Micro-Tom, a reference system for the Solanaceae genomics. BMC Genom. 2010, 11, 210-226. [CrossRef]

41. Boulos, C.; France, D.; Shengyi, L.; Parkin, I.A.P.; Haibao, T.; Xiyin, W.; Julien, C.; Harry, B.; Chaobo, T.; Birgit, S. Plant genetics. Early allopolyploid evolution in the post-Neolithic Brassica napus oilseed genome. Science 345, 950-953.

42. Yu, J.; Wang, J.; Lin, W.; Li, S.; Li, H.; Zhou, J.; Ni, P.; Dong, W.; Hu, S.; Zeng, C. The Genomes of Oryza sativa: A history of duplications. PLoS Biol. 2005, 3, e38-e54. [CrossRef] [PubMed]

43. Zapata, L.; Jia, D.; Willing, E.M.; Hartwig, B.; Bezdan, D.; Jiao, W.B.; Patel, V.; James, G.V.; Koornneef, M.; Ossowski, S. Chromosome-level assembly of Arabidopsis thaliana Ler reveals the extent of translocation and inversion polymorphisms. Proc. Natl. Acad. Sci. USA 2016, 113, E4052-E4060. [CrossRef] [PubMed]

44. Schmutz, J.; Cannon, S.B.; Schlueter, J.; Ma, J.; Mitros, T.; Nelson, W.; Hyten, D.L.; Song, Q.; Thelen, J.J.; Cheng, J. Genome sequence of the palaeopolyploid soybean. Nature 2010, 463, 178-183. [CrossRef] [PubMed]

45. Xie, T.; Chen, C.; Li, C.; Liu, J.; Liu, C.; He, Y. Genome-wide investigation of WRKY gene family in pineapple: Evolution and expression profiles during development and stress. BMC Genom. 2018, 19, 490-508. [CrossRef] [PubMed]

46. Cao, Z.H.; Zhang, S.Z.; Wang, R.K.; Zhang, R.F.; Hao, Y.J. Genome Wide Analysis of the Apple MYB Transcription Factor Family Allows the Identification of MdoMYB121 Gene Confering Abiotic Stress Tolerance in Plants. PLoS ONE 2013, 8, e69955-e69968. [CrossRef]

47. Wu, J.; Wang, Z.; Shi, Z.; Zhang, S.; Ming, R.; Zhu, S.; Khan, M.A.; Tao, S.; Korban, S.S.; Wang, H. The genome of the pear (Pyrus bretschneideri Rehd.). Genome Res. 2013, 23, 396-408. [CrossRef]

48. Lynch, M.; Conery, J.S. The evolutionary fate and consequences of duplicate genes. Science 2000, 290, 1151-1155. [CrossRef]

49. Otto, S.P.; Yong, P. The evolution of gene duplicates. Adv. Genet. 2002, 46, 451-483.

50. Duarte, J.M.; Liying, C.; P Kerr, W.; Qing, Z.; Xiaohong, Z.; Jim, L.M.; Hong, M.; Naomi, A.; Depamphilis, C.W. Expression pattern shifts following duplication indicative of subfunctionalization and neofunctionalization in regulatory genes of Arabidopsis. Mol. Biol. Evol. 2006, 23, 469-478. [CrossRef]

51. Hou, D.; Zhanchao, C.; Lihua, X.; Xiangyu, L.; Juan, L.; Shaohua, M.; Jian, G. The R2R3MYB Gene Family in Phyllostachys edulis: Genome-Wide Analysis and Identification of Stress or Development-Related R2R3MYBs. Front. Plant Sci. 2018, 9, 738-760. [CrossRef] [PubMed]

52. Levy, D.; Veilleux, R.E. Adaptation of potato to high temperatures and salinity-a review. Am. J. Potato Res. 2007, 84, 487-506. [CrossRef]

53. Arvin, M.J.; Donnelly, D.J. Screening potato cultivars and wild species to abiotic stresses using an electrolyte leakage bioassay. J. Agric. Sci. Technol. 2008, 10, 33-42.

54. Trudi, H.; Feng, L.S.; Parish, R.W. AtMYB103 regulates tapetum and trichome development in Arabidopsis thaliana. Plant J. 2010, 35, 177-192.

55. Rumi, T.; Mineko, I.; Kiyotaka, O.; Takuji, W. Functional analysis of the epidermal-specific MYB genes CAPRICE and WEREWOLF in Arabidopsis. Plant Cell 2007, 19, 2264-2277. 
56. Frank, M.; Harald, K.; Pawel, B.; Bernd, W. The Arabidopsis transcription factor MYB12 is a flavonol-specific regulator of phenylpropanoid biosynthesis. Plant Physiol. 2005, 138, 1083-1096.

57. Teng, S.; Keurentjes, J.; Bentsink, L.N.; Koornneef, M.; Smeekens, S. Sucrose-Specific Induction of Anthocyanin Biosynthesis in Arabidopsis Requires the MYB75/PAP1 Gene. Plant physiol. 2006, 139, 1840-1852. [CrossRef]

58. Yuan-Yuan, L.; Ke, M.; Cheng, Z.; Xian-Yan, Z.; Hua-Lei, Z.; Huai-Rui, S.; Yu-Jin, H. MdCOP1 ubiquitin E3 ligases interact with MdMYB1 to regulate light-induced anthocyanin biosynthesis and red fruit coloration in apple. Plant Physiol. 2012, 160, 1011-1022.

59. Hiroshi, A.; Takeshi, U.; Takuya, I.; Motoaki, S.; Kazuo, S.; Kazuko, Y.S. Arabidopsis AtMYC2 (bHLH) and AtMYB2 (MYB) function as transcriptional activators in abscisic acid signaling. Plant Cell 2003, 15, 63-78.

60. Nagaoka, S.; Takano, T. Salt tolerance-related protein STO binds to a MYB transcription factor homologue and confers salt tolerance in Arabidopsis. J. Exp. Bot. 2003, 54, 2231-2237. [CrossRef]

61. Yao, L.; Jiang, Y.; Lu, X.; Wang, B.; Zhou, P.; Wu, T. A R2R3-MYB transcription factor from Lablab purpureus induced by drought increases tolerance to abiotic stress in Arabidopsis. Mol. Biol. Rep. 2016, 43, 1089-1100. [CrossRef] [PubMed]

62. Jin, H.; Cominelli, E.; Bailey, P.; Parr, A.; Mehrtens, F.; Jones, J.; Tonelli, C.; Weisshaar, B.; Martin, C. Transcriptional repression by AtMYB4 controls production of UV-protecting sunscreens in Arabidopsis. EMBO J. 2014, 19, 6150-6161. [CrossRef] [PubMed]

63. Preston, J.; Wheeler, J.J.; Li, S.; Parish, R. AtMYB32 is required for normal pollen development in Arabidopsis thaliana. Plant J. 2010, 40, 979-995. [CrossRef] [PubMed]

64. Silvia, F.; Eric, L.; Salazar-Henao, J.E.; Pedro, F.N.; Joan, R.; David, C.R. AtMYB7, a new player in the regulation of UV-sunscreens in Arabidopsis thaliana. Plant Cell Physiol. 2014, 55, 507-516.

65. Cominelli, E.; Galbiati, M.; Vavasseur, A.; Conti, L.; Sala, T.; Vuylsteke, M.; Leonhardt, N.; Dellaporta, S.L.; Tonelli, C. A Guard-Cell-Specific MYB Transcription Factor Regulates Stomatal Movements and Plant Drought Tolerance. Curr. Biol. 2005, 15, 1196-1200. [CrossRef] [PubMed]

66. Seo, P.J.; Xiang, F.; Qiao, M.; Park, J.Y.; Lee, Y.N.; Kim, S.G.; Lee, Y.H.; Park, W.J.; Park, C.M. The MYB96 transcription factor mediates abscisic acid signaling during drought stress response in Arabidopsis. Plant Physiol. 2009, 151, 275-289. [CrossRef] [PubMed]

67. Seo, P.J.; Park, C.M. MYB96-mediated abscisic acid signals induce pathogen resistance response by promoting salicylic acid biosynthesis in Arabidopsis. New Phytol. 2010, 186, 471-483. [CrossRef] [PubMed]

68. Reyes, J.; Chua, N. ABA induction of miR159 controls transcript levels of two MYB factors during Arabidopsis seed germination. Plant J. 2010, 49, 592-606. [CrossRef]

69. Manu, A.; Yujin, H.; Avnish, K.; Chun-Hai, D.; Hiroaki, F.; Xianwu, Z.; Jian-Kang, Z. A R2R3 type MYB transcription factor is involved in the cold regulation of $\mathrm{CBF}$ genes and in acquired freezing tolerance. J. Biol. Chem. 2006, 281, 37636-37645.

70. Mengiste, T.; Chen, X.; Salmeron, J.; Dietrich, R. The BOTRYTIS SUSCEPTIBLE1 Gene Encodes an R2R3MYB Transcription Factor Protein That Is Required for Biotic and Abiotic Stress Responses in Arabidopsis. Plant Cell 2003, 15, 2551-2565. [CrossRef]

71. Jung, C.; Seo, J.S.; Han, S.W.; Koo, Y.J.; Kim, C.H.; Song, S.I.; Nahm, B.H.; Choi, Y.D.; Cheong, J.J. Overexpression of AtMYB44 enhances stomatal closure to confer abiotic stress tolerance in transgenic Arabidopsis. Plant Physiol. 2008, 146, 623-635. [CrossRef] [PubMed]

72. Edgar, R. MUSCLE: Multiple sequence alignment with high accuracy and high throughput. Nucleic Acids Res. 2004, 32, 1792-1797. [CrossRef] [PubMed]

73. Kumar, S.; Stecher, G.; Tamura, K. MEGA7: Molecular Evolutionary Genetics Analysis version 7.0 for bigger datasets. Mol. Biol. Evol. 2016, 33, 1870-1874. [CrossRef] [PubMed]

74. Krzywinski, M.; Schein, J.I. Circos: An information aesthetic for comparative genomics. Genome Res. 2009, 19, 1639-1645. [CrossRef] [PubMed]

75. Wang, Y.; Tang, H.; DeBarry, J.D.; Tan, X.; Li, J.; Wang, X. MCScanX: A toolkit for detection and evolutionary analysis of gene synteny and collinearity. Nucleic Acids Res. 2012, 40, e49. [CrossRef] [PubMed]

76. Liu, C.; Xie, T.; Chen, C.; Luan, A.; Long, J.; Li, C.; Ding, Y.; He, Y. Genome-wide organization and expression profiling of the R2R3-MYB transcription factor family in pineapple (Ananas comosus). BMC Genom. 2017, 18, 503-519. [CrossRef] [PubMed] 
77. Nicot, N.; Hausman, J.F.; Hoffmann, L.; Evers, D. Reference gene selection for RT-qPCRnormalization in potato during biotic and abiotic stress. J. Exp. Bot. 2005, 56, 2907-2914. [CrossRef] [PubMed]

78. Livak, K.J.; Schmittgen, T.D. Analysis of relative gene expression data using real-time quantitative PCR and the 2(-Delta Delta C(T)) Method. Methods 2012, 25, 402-408. [CrossRef]

(C) 2019 by the authors. Licensee MDPI, Basel, Switzerland. This article is an open access article distributed under the terms and conditions of the Creative Commons Attribution (CC BY) license (http://creativecommons.org/licenses/by/4.0/). 